\title{
Molecular determinants of response to high-dose androgen therapy in prostate
} \section{cancer}

\author{
Michael D. Nyquist, ${ }^{1}$ Alexandra Corella, ${ }^{1}$ Osama Mohamad, ${ }^{2}$ Ilsa Coleman, ${ }^{1}$ Arja Kaipainen, ${ }^{1}$ \\ Daniel A. Kuppers, ${ }^{1}$ Jared M. Lucas, ${ }^{1}$ Patrick J. Paddison, ${ }^{1}$ Stephen R. Plymate, ${ }^{3,4}$ Peter S. Nelson, ${ }^{1,3}$ \\ and Elahe A. Mostaghel ${ }^{1,3,4}$ \\ ${ }^{1}$ Fred Hutchinson Cancer Research Center, Seattle, Washington, USA. ${ }^{2}$ Faculty of Medicine, Benha University, Benha, \\ Egypt. ${ }^{3}$ Department of Medicine, University of Washington, Seattle, Washington, USA. ${ }^{4}$ Geriatric Research, Education, and \\ Clinical Center, VA Puget Sound Health Care System, Seattle, Washington, USA.
}

Clinical trials of high-dose androgen (HDA) therapy for prostate cancer (PC) have shown promising efficacy but are limited by lack of criteria to identify likely responders. To elucidate factors that govern the growth-repressive effects of HDAs, we applied an unbiased integrative approach using genetic screens and transcriptional profiling of PC cells with or without demonstrated phenotypic sensitivity to androgen-mediated growth repression. Through this comprehensive analysis, we identified genetic events and related signaling networks that determine the response to both HDA and androgen withdrawal. We applied these findings to develop a gene signature that may serve as an early indicator of treatment response and identify men with tumors that are amenable to HDA therapy.

Conflict of interest: The authors have declared that no conflict of interest exists.

Copyright: () 2019, American Society for Clinical Investigation.

Submitted: April 22, 2019

Accepted: September 4, 2019

Published: October 3, 2019

Reference information: /CI Insight. 2019;4(19):e129715.

https://doi.org/10.1172/jci.

insight.129715.

\section{Introduction}

Prostate cancer (PC) is driven by androgen receptor (AR) signaling. Androgen deprivation therapy (ADT) is the frontline treatment for metastatic PC. Although ADT is initially effective at suppressing growth of PC, it inevitably fails after 1 to 2 years, giving rise to castration-resistant PC (CRPC), in addition to being associated with significant quality of life complications (1). Targeting the AR program with next-generation AR-signaling inhibitors, such as enzalutamide (ENZ) or abiraterone, can be effective but responses are not durable and most men will progress within months $(2,3)$. CRPC continues to be driven primarily by AR signaling through diverse mechanisms that include $A R$ amplification, $A R$ mutations, intratumoral synthesis of androgens, and the production of ligand-independent AR splice variants (4).

AR signaling can be dichotomous, promoting growth at lower androgen levels while suppressing PC growth when hyperstimulated by high doses of androgen $(5,6)$. The observation that PC cells can adjust to too little or too much AR signaling over time by up- or down-tuning AR activity (7) has led to the development of an approach termed bipolar androgen therapy (BAT) in which periods of high-dose testosterone injections, given every 28 days, are cycled with periods of ADT, theoretically preventing cells from adapting to a static androgen environment while improving quality of life $(5,8)$. This approach has achieved response rates of $40 \%$ to $60 \%$ in clinical trials of patients with CRPC $(9,10)$. A recent study of BAT for ADT-naive PC patients met its primary endpoints for efficacy and indicated improved quality of life (10). In a phase II study of BAT in patients with CRPC who were previously treated with ENZ, findings showed BAT to be safe, effective, and able to resensitize $52 \%$ of patients with PC to ENZ (11). These trials and anecdotal reports of exceptional responses to BAT (12) highlight the potential of this therapy.

A diverse array of mechanisms have been proposed to explain the growth-repressive effects of high-dose androgen (HDA): (a) transcription of cell cycle-inhibitory genes like p16 and p21 (13-15); (b) transcriptional suppression of $M Y C, C D K 1$, and $B C L 2(16,17)$; (c) induction of DNA-damage via transcription stress induced by TOP2B-mediated double-strand DNA breaks $(6,18)$; (d) induction of DNA damage and senescence by the production of ROS via acute SRC-AKT activation (19, 20); (e) induction of ROS through AR-induced SMAD signaling (21); (f) induction of ROS and p21 in an mTOR-dependent manner (22); (g) prevention of replication relicensing through the stabilization of 
AR on DNA $(6,23)$; (h) androgen-induced expression of endoplasmic reticulum stress response genes such as NDRG1 $(24,25)$; and (i) RB1-dependent suppression of cell cycle-related genes like $M C M 2$, $M C M 7$, and FANCI (26). Despite these mechanistic insights, the genetic factors that mediate sensitivity or resistance to HDA are poorly understood and criteria for prospectively identifying responders or nonresponders to HDA-based therapies have not been established.

To identify factors that govern response to this treatment modality, we applied an integrative approach using genetic screens and RNA sequencing (RNA-seq) analysis to identify and functionally annotate genetic determinants mediating sensitivity and resistance. By characterizing the transcriptomic changes associated with low, proliferative, and high AR activities, we identified modalities of the AR-signaling program that governed growth and survival. In a clinical context, coordinated use of these gene expression signatures may identify CRPCs that are likely to respond to AR-directed therapies.

\section{Results}

Androgen-mediated suppression of PC growth. To investigate the molecular determinants of response and resistance to HDA, we first characterized the responses of PC models to dose ranges of androgen. Under normal growth conditions (10\% FBS), increasing androgen concentrations potently repressed the growth of LNCaP (Figure 1A), VCaP (Figure 1B), 22PC (Figure 1C), and LAPC4 (Figure 1D) starting around $10 \mathrm{pM}$ R1881, whereas R1AD1 (Figure 1E) and 22Rv1 (Figure 1F) were essentially resistant to the growth-modulating effects of androgen. Charcoal-stripped serum (CSS), in which steroidal compounds and cytokines such as IGF are depleted (27), is routinely used to mimic low-androgen conditions and investigate the effects of HDA in vitro $(6,18)$. LNCaP (Figure $1 \mathrm{G}$ ) and VCaP cells (Figure 1H) displayed a biphasic growth response when treated with R1881 in CSS media, with maximal proliferation induced at $100 \mathrm{pM}$ R1881 and less proliferation induced by doses over $1 \mathrm{nM}$. R1AD1 and 22Rv1 cells treated with androgen in CSS displayed a weak biphasic response (Supplemental Figure 1, A and B; supplemental material available online with this article; https://doi.org/10.1172/jci.insight.129715DS1). However, in CSS media, no dose of androgen suppressed viability below baseline, suggesting experiments performed in CSS inadequately model the growth suppressive effects of androgen. Furthermore, FBS-containing medium represents a more physiological environment, with androgen levels similar to those observed in patients with CRPC (28), and thus may be more appropriate for molecular studies evaluating the phenotype and mechanism of androgen-mediated growth suppression.

Independent regulation of mitotic and biosynthetic AR functions. To characterize the transcriptional changes caused by HDA, we performed RNA-seq analysis comparing 3 phenotypically defined androgen-signaling-induced growth states (all in 10\% FBS containing media at 48 hours): (a) growth suppression in response to $\mathrm{ADT}(10 \mu \mathrm{M}$ ENZ); (b) proliferation under normal growth conditions (vehicle control); and (c) growth suppression in response to HDA (10 nM R1881) (Supplemental Figure 2A). We observed substantial overlap in genes regulated by HDA versus vehicle across the 4 cell lines (FDR < 0.05), with 1626 genes upregulated (Supplemental Figure 2B) and 1667 genes downregulated (Supplemental Figure 2C) between VCaP, 22PC, LNCaP, and LAPC4. Gene set enrichment analysis (GSEA) (29) revealed positive enrichments of Hallmark gene sets associated with canonical AR functions and luminal differentiation (e.g., Androgen_response, Adipogenesis, and Cholesterol_homeostasis), and suppression of gene sets related to proliferation and cell cycle (e.g., E2F_targets, DNA_repair, G2/M_checkpoint, Mitotic_spindle, and MYC_targets_V1/2; Figure 2A). Fewer genes were significantly regulated by ENZ across all 4 cell lines, with 384 genes upregulated (Supplemental Figure 2D) and 679 genes downregulated (Supplemental Figure $2 \mathrm{E})$. As expected, inhibition of AR activity with ENZ strongly downregulated gene sets related to canonical AR signaling, as well as gene sets associated with cell cycle and proliferation (Figure $2 \mathrm{~B}$ ).

To define the molecular signaling underlying ADT- and HDA-mediated growth repression, we identified genes that were concordantly repressed (termed biphasic genes, Figure 2C, blue) or concordantly induced (termed inverse-biphasic, Figure 2C, red) in response to both ENZ-mediated ADT and R1881-mediated HDA. We also identified genes that were oppositely regulated by ENZ and R1881, including AR-induced genes (Figure 2C, green) with decreased expression at low AR activity and increased expression at high AR activity, and AR-repressed genes (Figure 2C, yellow) with increased expression at low AR activity and decreased expression at high AR activity. Genes with biphasic expression comprised the largest subset, with 288 genes (66\%) similarly regulated across all 4 cell lines (LNCaP, 22PC-EP, VCaP, LAPC4) (Figure 2D, Supplemental Figure 2F, and Supplemental Table 1). AR-induced genes comprised the next largest 
A
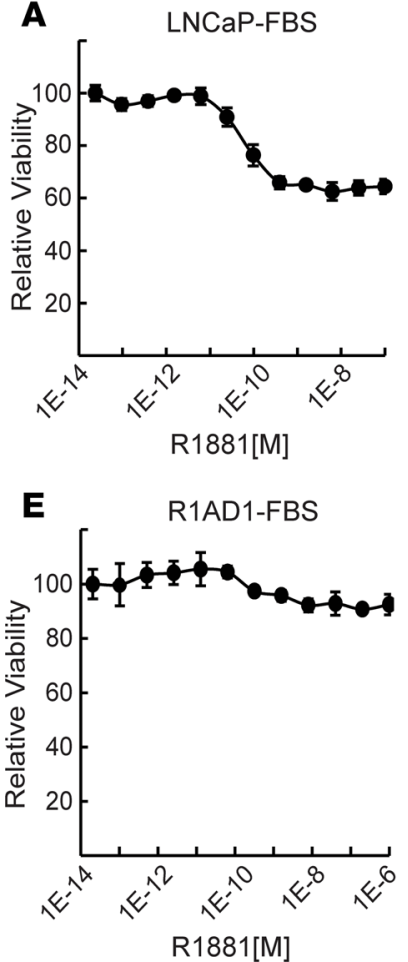

B

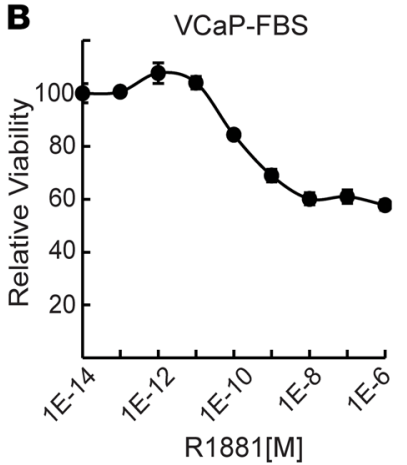

$\mathbf{F}$

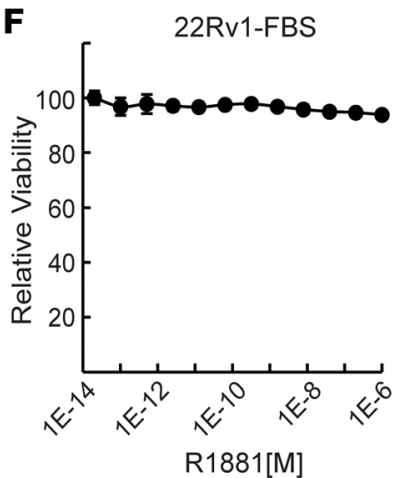

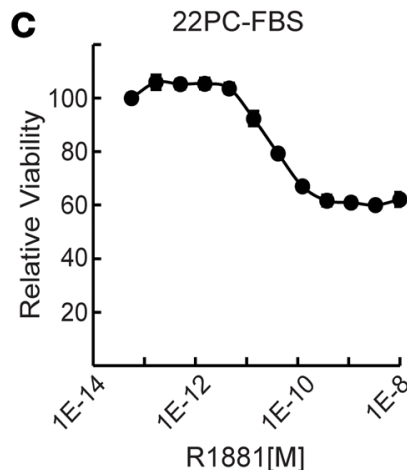

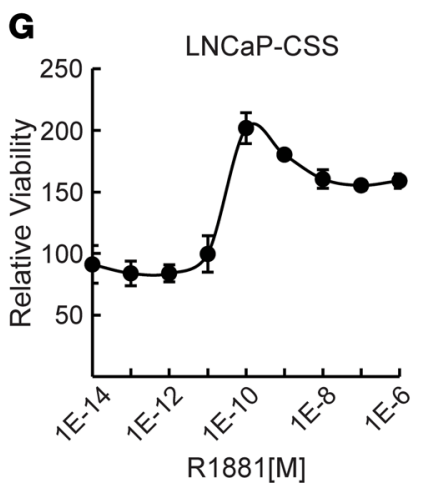

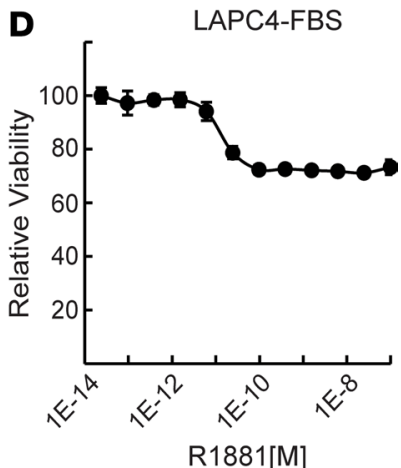

H

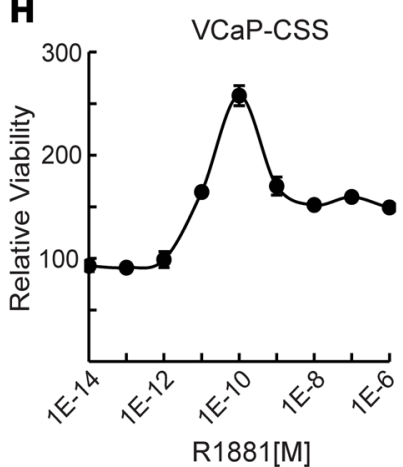

Figure 1. Suppression of prostate cancer viability by androgens. Relative viability (Celltiter-glo) of PC cell lines was measured in response to a dose-range of the androgen R1881 ( $n=4)$ in normal growth media (10\% FBS) for (A) LNCaP (B) VCaP (C) 22PC-EP, (D) LAPC4, (E) R1AD1, and (F) 22Rv1. Dose-responses to R1881 in $10 \%$ CSS media after 5 days in culture for (C) LNCaP and (H) VCaP.

category with 66 genes (15\%). When the stringencies were relaxed to include genes that were commonly regulated in 3 out of 4 cell lines, the number of genes in the biphasic subset increased to 608 and in the AR-induced subset increased to 436 (Supplemental Figure 2G and Supplemental Table 1).

Pathway analysis using the PANTHER gene ontology (GO) (30) overrepresentation test revealed that each of these gene sets mediated distinct biologic functions. Consistent with the GSEA findings discussed previously (Figure 2, A and B), biphasic genes were associated with proliferation and DNA replication, with pathway enrichments for cell cycle, mitosis, chromosome segregation, DNA-metabolizing pathways including de novo pyrimidine deoxyribonucleotide synthesis, and DNA replication (Figure 2E). The inverse-biphasic category of genes did not include any statistically overrepresented pathways across all 4 cell lines, but in 3 of 4 cell lines the data showed that pathways relating to PDGF signaling and transcription factor activity were enriched (Figure $2 \mathrm{~F}$ ). This category also included notable PC-related transcription factors NCOA3 and FOXO3 (Supplemental Table 1). AR-induced genes consisted of metabolic and biosynthetic programs, such as lipid and cholesterol biosynthesis, as well as glycolysis and gluconeogenesis (Figure 2G). Of note, AR activity drives lipid biosynthetic programs largely through the transcription factor SREBF1 (31, 32), and SREBF targets were overrepresented in the AR-induced gene set. AR-repressed genes were involved in the regulation of protein translation and ribosome biosynthesis (Figure $2 \mathrm{H}$ ). Notably, GATA2, a known effector of castration resistance, was represented in the AR-repressed subset $(33,34)$. These data suggest that not all AR-responsive genes are linearly AR-induced or AR-repressed, and that each subset of AR-regulated genes associates with distinct biologic functions. For example, metabolic and biosynthetic functions may be regulated independently from cell-cycle and proliferative functions.

Enrichment of cell-cycle drivers in overexpression screen for HDA resistance. Although RNA-seq studies provide a global picture of the effects of AR signaling on cellular pathways involved in regulating the growth-repressed phenotypes, they do not provide functional information on specific genetic mechanisms. We therefore pursued an unbiased functional genetics approach employing whole-genome overexpression and knockdown screens to identify molecular pathways functionally relevant to the growth-repressed phenotype and determined the overlap of these findings with the signaling pathways identified in the RNA-seq 
A Adipogenesis HDA vs Vehicle

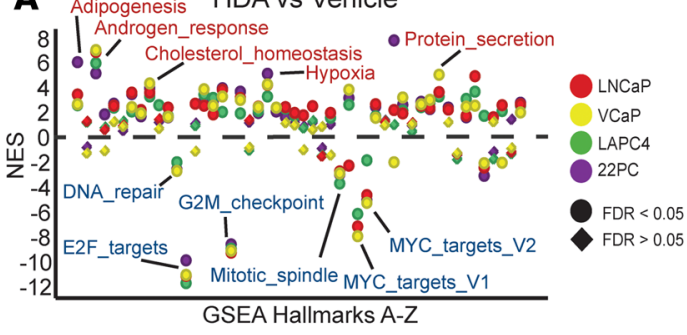

B
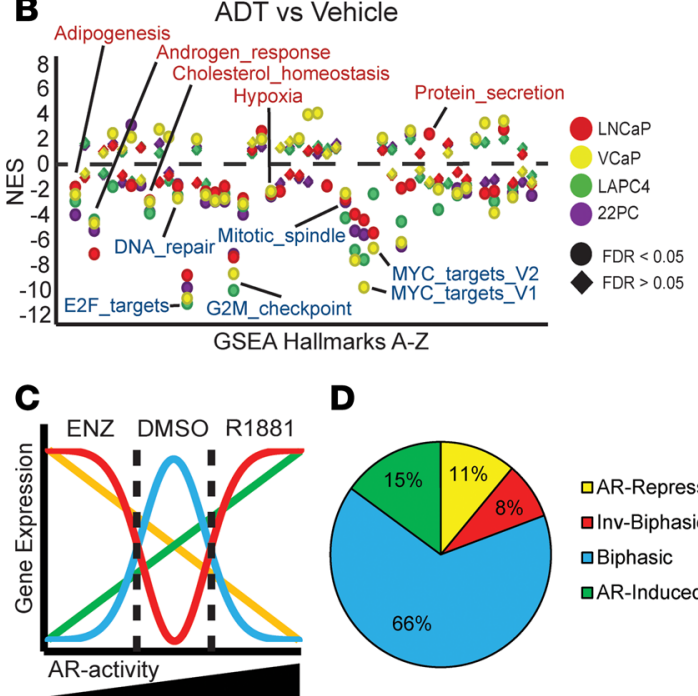

D

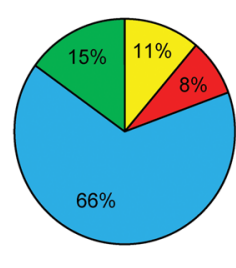

$\mathbf{E}$

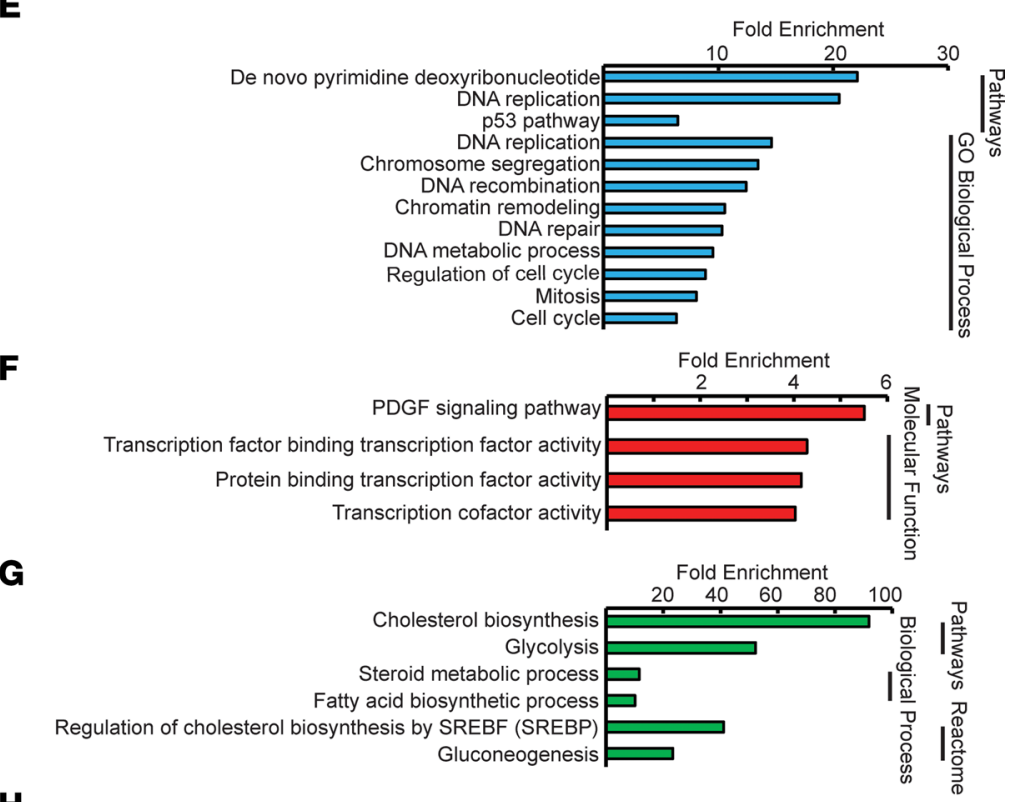

F

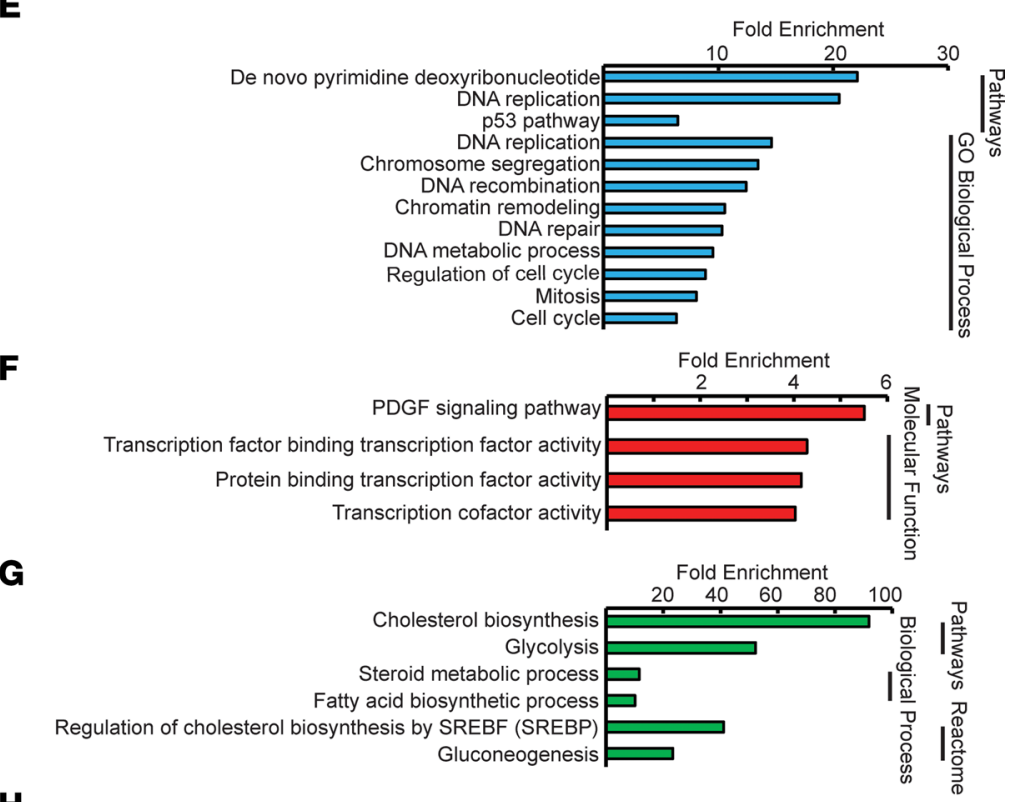

G

Fold Enrichment

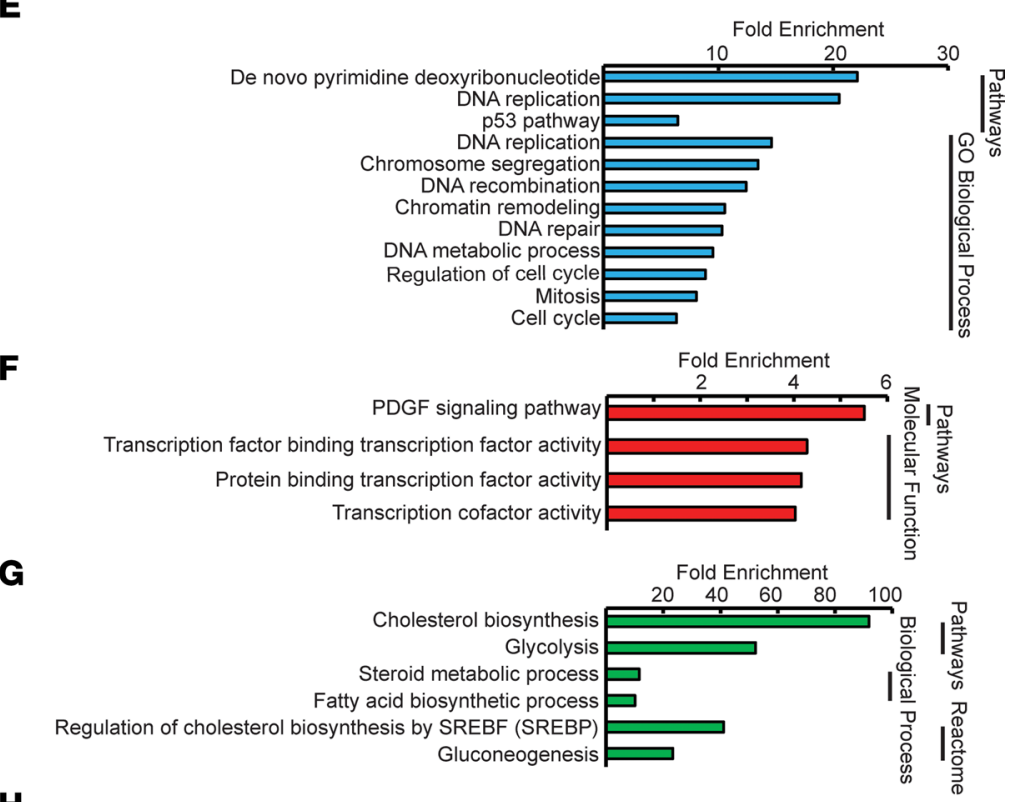

H
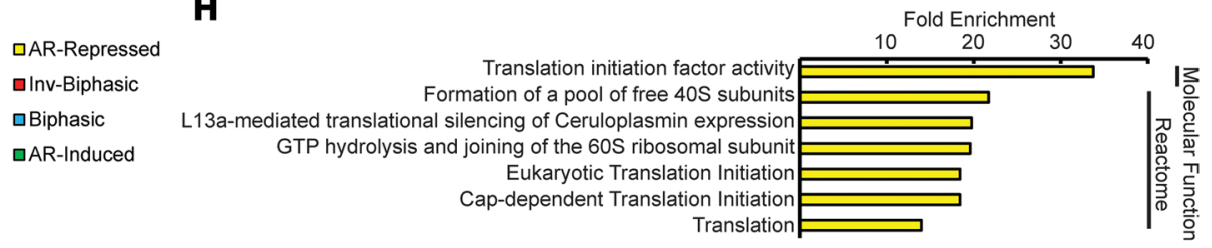

Figure 2. The androgen receptor signaling program is comprised of multiple modalities. (A) Normalized-enrichment scores (NES) of GSEA Hallmark gene sets for all 4 cell lines. Significant gene sets comparing $10 \mathrm{nM}$ R1881 versus vehicle (FDR $<0.05)$ are marked as circles. Androgen receptor-related gene sets are labeled in red, cell cycle-related gene sets in blue $(n=2)$. (B) Same as A for $10 \mu \mathrm{M}$ ENZ-related gene expression changes. (C) AR-signaling modalities are diagrammed: biphasic (blue), inverse-biphasic (red), AR-repressed (yellow), AR-induced (green). (D) Percentage of AR-responsive genes in each category. Gene ontology (GO) pathways that are overrepresented in the (E) biphasic gene subset, (F) inverse-biphasic subset, (C) AR-induced subset, and (H) AR-repressed subset. ADT, androgen deprivation therapy; AR, androgen receptor; GSEA, gene set enrichment analysis; HDA, high-dose androgen.

analysis. We performed a whole-genome ORF library screen on LNCaP cells under conditions of HDA. LNCaPs were transduced with the ORF library and cultured in normal growth media with vehicle or 10 nM R1881 for 25 days, at which time DNA was harvested and sequenced (primers in Supplemental Table 2). Barcode read-counts at the end of the 25 days were compared with day 0 to determine depletion or enrichment of the associated ORF. Overexpression of genes in several signaling programs mediated resistance to HDA, including (a) cell-cycle factors that govern the G1/S transition, such as D-type cyclins and their binding partners $C D K 4$ and $C D K 6$; (b) modulators of AR signaling, such as PIAS1 and FGFR1; and (c) stem cell-related and reprogramming factors such as SOX2, KLF4, LIN28A, and WT1 (Figure 3A).

Given that amplification and overexpression of factors that induce cell cycle are common events in CRPC (35), and that these factors regulate the same cellular processes as the biphasic genes associated with the growth repressive phenotype, $C D K 4 / 6$ and D-type cyclins were chosen for further validation in competitive enrichment assays (Figure 3B). Because ADT and HDA both arrest cells in the G1 phase of the cell cycle (23), we hypothesized that cyclin D and $C D K 4 / 6$ overexpression should mediate resistance to both ENZ and R1881. A competition assay with the 2 empty vector controls established that the fluorescent proteins themselves did not impart a selective advantage (Supplemental Figure 3, A and B). Overexpression of cyclin D1 (CCND1), cyclin D2 (CCND2), and cyclin D3 (CCND3) were strongly enriched in LNCaPs in response to R1881 and ENZ and modestly enriched in VCaPs (Figure 3C). Overexpression of CDK4 and CKD6 similarly promoted growth in LNCaPs in response to R1881 and ENZ, with more modest enrichment in VCaPs. These results demonstrate that increased expression of cell-cycle drivers can override AR-mediated control of proliferation and implicate processes associated with biphasic genes as primary mediators of response to ADT and HDA. 


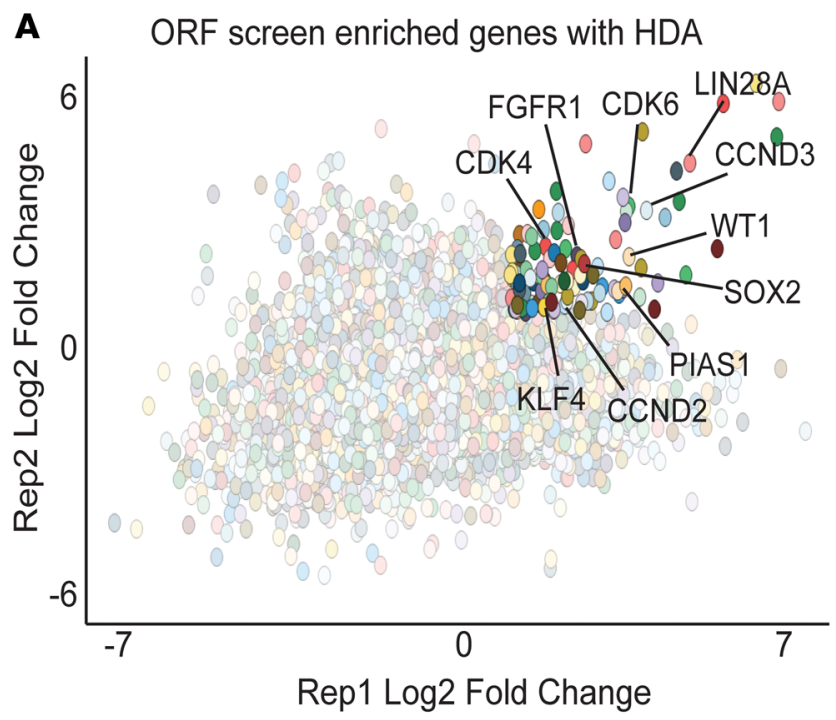

B

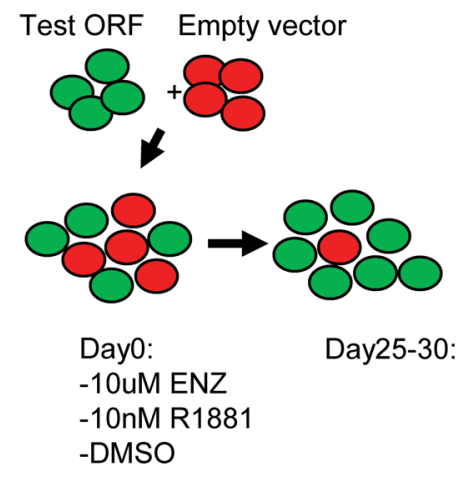

C

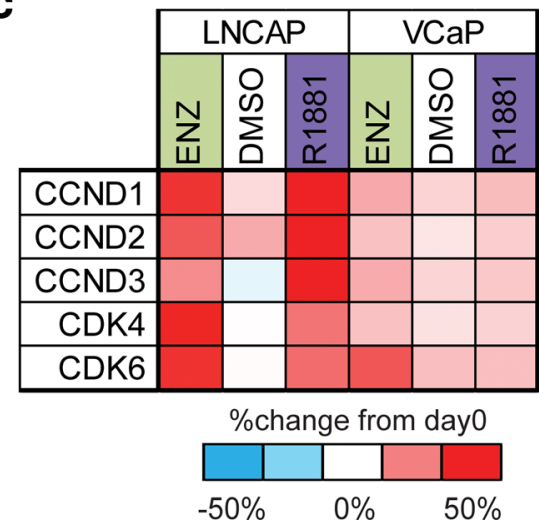

Figure 3. ORF screen identifies cell-cycle regulator upregulation as a mechanism of dual resistance. (A) Log2 fold change in frequency for 2 biological replicates of an ORF screen for genes that modulate responses to $10 \mathrm{nM}$ R1881 in LNCaP. (B) Competition assay schematic to measure the selective advantage or disadvantage of gene. (C) Heatmap of competition assays that validated screen hits.

Enrichment of cell-cycle inhibitors in whole-genome CRISPR/CAS9 KO screens for HDA sensitivity. To complement the ORF screen, we performed whole-genome CRISPR/CAS9 KO screens to identify genes required for response to AR-mediated growth repression. LNCaP cells were transduced with a pooled whole-genome sgRNA library encoding 10 sgRNAs per gene (36), cultured in normal growth media with vehicle, $10 \mu \mathrm{M}$ ENZ, or $10 \mathrm{nM}$ R1881 for 25 days, and sequenced to identify the sgRNAs enriched or depleted in the ENZ- or HDA-treated cell populations. Genes associated with enriched sgRNAs (in which knockdown promoted cell growth) or depleted sgRNAs (in which knockdown promoted cell loss) were determined by MAGECK analysis $(37,38)$. We performed 4 biological replicates and determined overlap between the top 2000 scoring genes for enrichment and depletion across all 4 replicates.

In agreement with the pathways identified by the RNA-seq studies and the ORF screen, sgRNAs targeting critical drivers of cell-cycle progression and mitosis, such as CCNA2, CCND1, CDK2, CDK4, CDC20/27/-45, and $M Y C$, decreased growth and were depleted regardless of treatment condition (Figure 4A). Of note, SOX4, which positively regulates plasticity factor EZH2 (39) and oncogenic AKT signaling (40), was depleted in all 4 replicates of the HDA treatment but not the vehicle or ADT conditions. sgRNAs that target tumor suppressors that inhibit cell-cycle progression in response to stress, such as CDKN-family genes, GADD45G, and CHEK2, were enriched under HDA and ADT conditions (Figure 4A). These targets were validated in competitive enrichment assays using separate, design-optimized sgRNAs (Supplemental Figure $3 C$ and Supplemental Table 2). KOs of CDKN1A, CDKN1B, CDKN1C, CDKN2B, GADD45G, and CHEK2 in LNCaP cells were selected in multiple conditions but most strongly in the presence of R1881 (Figure 4B). 
In VCaP cells, loss of $C D K N 1 B, C D K N 2 A$, and $C H E K 2$ were modestly enriched in multiple conditions, whereas loss of $C D K N 1 C, C D K N 2 B$, and $G A D D 45 G$ were not strongly selected for (Figure $4 \mathrm{~B}$ ).

Loss of TP53 was enriched in all treatment conditions in both cell models (Figure 4B). Interestingly, $R B 1 \mathrm{KO}$ did not influence response to R1881 in LNCaP, whereas loss of $R B 1$ was enriched in VCaP cells (Figure 4B). Of note, VCaP cells are heterozygous for mutant TP53 (41), and TP53 loss can synergize with $R B 1$ loss to promote tumorigenesis (42). To determine whether TP53 loss is necessary for RB1 loss to mediate resistance to HDA in LNCaP, we performed a dual competitive enrichment assay wherein LNCaP cells were transduced with sgRNAs targeting one, both, or neither of TP53 and RB1 and treated with 10 nM R1881. Dual loss of TP53 and RB1 was the most strongly enriched (Figure 4C, from 19\% to 45\%), suggesting therapy resistance may be a result of multiple synergistic events. Overall, these data demonstrate that loss of cell-cycle inhibitors and tumor suppressors uncouple both high- and low-dose AR signaling from control of proliferation, suggesting a mechanism by which resistance to HDA and ADT may be co-acquired during cancer progression.

Functional annotation of AR-regulated genes through targeted CRISPR/CAS9 screens. Although sensitivity to ADT and HDA may be influenced by loss or gain of cell cycle-related tumor suppressors and oncogenes, respectively, it is unclear which specific AR-regulated genes are mediators of growth suppression by HDA. To identify these genes, we performed a focused CRISPR/CAS9 screen consisting of AR-responsive genes in LNCaP cells under $10 \mathrm{nM}$ R1881 conditions. Targeted screens allow specific components of the transcriptome to be examined in isolation at greater sgRNA-per-gene depth and read coverage. Moreover, by removing genes essential for survival that do not change with androgens, AR-responsive genes that are critical for growth and survival can be identified with higher confidence and less noise (Supplemental Table 3).

In agreement with the whole-genome CRISPR screen, sgRNAs mediating loss of AR-regulated stress-response genes, such TP53, CDKN1A, and GADD45G, countered the growth repressive effect of HDA and were strongly enriched in response to R1881 (Figure 5A). sgRNAs targeting tumor suppressors PPP1R15A and NDRG4 were also enriched (Figure 5A). In contrast, sgRNAs targeting AR-regulated genes involved in promoting PC growth and progression were strongly depleted, including GATA2, SOX9, BRCA1, EZH2, BARD1, BCL2L1, FOXA1, WEE1, and MYC (Figure 5B).

The RNA-seq studies described previously demonstrated that tumor suppressor genes, which were enriched by knockdown in the CRISPR screens, were largely upregulated at the transcript level by HDA (Figure 5C, top). In contrast, with the exceptions of $S O X 9$ and BCL2L1, genes that supported growth and survival, which were depleted by knockdown in the CRISPR screen, were largely downregulated by HDA (Figure 5C, bottom).

Underlining their functional importance, biphasically regulated genes were strongly clustered among the most depleted of all AR-responsive genes (Figure 5E). Compared with the median rank of 1865, the biphasic genes were more highly represented among the top ranked/most depleted genes with a median rank of 422 and 316 in each replicate, respectively, supporting the functional relevance of this gene set for the growth-repressive effects of HDA and ADT (Figure 5D). These findings suggest that AR exerts control over the proliferation of PC through multiple factors that act in parallel, including both upregulation of tumor suppressors and suppression of oncogenes and biphasic genes.

Suppression of biphasic gene expression is an innate function of $A R$. A number of the molecular mechanisms proposed for AR-mediated growth suppression rely on the premise that CRPC cells have uncontrolled growth and are thus more susceptible to the transcriptional (18), mitochondrial oxidative (19-21), or mitotic stresses $(6,23)$ induced by acute doses of androgen. However, AR signaling has been previously reported to arrest the growth of various untransformed cell types, including prostate epithelial cells (43). To determine whether AR signaling suppresses biphasic gene expression in untransformed cells in a manner similar to that observed in PC cells, we used the hTERT-immortalized prostate epithelial cell (PREC) line 957E/ TERT-AR that ectopically expresses AR (43). These cells were treated with $1 \mathrm{nM}$ R1881 or vehicle control for 24 hours and analyzed by RNA-seq. Androgen strongly repressed the biphasic gene set signature and induced the inverse-biphasic gene signature (Figure 6A). Among the top 250 genes repressed by androgen were 89 biphasic genes, such as FOXM1, RAD54L, E2F1, and CCNA2 (Figure 6A and Supplemental Table 4). Inverse-biphasic genes, such as FOXO3, MXD4, and $S O S 2$, were among the top 250 upregulated genes, as were AR-targets $M A F$ and FKBP5 (Figure 6A and Supplemental Table 5). As in the cancer cells, biphasic genes partially overlap with $E 2 F 1$ and $M Y C$ targets (Figure 6A). These data suggest that AR-mediated growth suppression is achieved through transcriptional repression of cell-cycle gene expression in both untransformed and transformed cells. 
A

\begin{tabular}{|c|c|c|c|c|c|c|}
\hline$\sum_{i}^{\vec{D}}$ & $\begin{array}{l}+ \\
0 \\
\stackrel{\lambda}{\pi} \\
0 \\
\text { s } \\
\frac{1}{d} \\
>\end{array}$ & 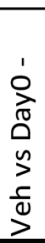 & 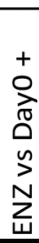 & 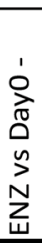 & 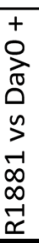 & 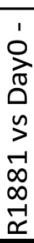 \\
\hline AURKA & 0 & 4 & 0 & 3 & 0 & 2 \\
\hline CCNA2 & 0 & 4 & 0 & 2 & 0 & 3 \\
\hline CCND1 & 0 & 3 & 0 & 3 & 0 & 2 \\
\hline CCNE2 & 0 & 0 & 0 & 0 & 0 & 2 \\
\hline $\mathrm{CDC} 20$ & 0 & 4 & 0 & 3 & 0 & 3 \\
\hline $\mathrm{CDC} 27$ & 0 & 3 & 0 & 1 & 0 & 1 \\
\hline CDC45 & 0 & 3 & 0 & 1 & 0 & 4 \\
\hline CDK2 & 0 & 2 & 0 & 0 & 0 & 3 \\
\hline CDK4 & 0 & 3 & 0 & 1 & 0 & 1 \\
\hline MYC & 0 & 4 & 0 & 3 & 0 & 3 \\
\hline SF1 & 0 & 4 & 0 & 4 & 0 & 3 \\
\hline SOX4 & 0 & 1 & 0 & 0 & 0 & 4 \\
\hline CDKN1B & 0 & 1 & 2 & 0 & 2 & 0 \\
\hline CDKN2A & 0 & 0 & 2 & 0 & 2 & 0 \\
\hline CDKN2B & 0 & 0 & 1 & 1 & 1 & 0 \\
\hline CDKN2C & 0 & 0 & 1 & 0 & 2 & 0 \\
\hline CDKN3 & 2 & 0 & 1 & 0 & 1 & 0 \\
\hline CHEK2 & 1 & 0 & 3 & 0 & 2 & 0 \\
\hline GADD45B & 0 & 0 & 0 & 0 & 2 & 0 \\
\hline GADD45G & 0 & 0 & 0 & 0 & 3 & 0 \\
\hline RB1 & 1 & 0 & 1 & 0 & 2 & 0 \\
\hline TP53 & 4 & 0 & 1 & 0 & 1 & 1 \\
\hline
\end{tabular}

B

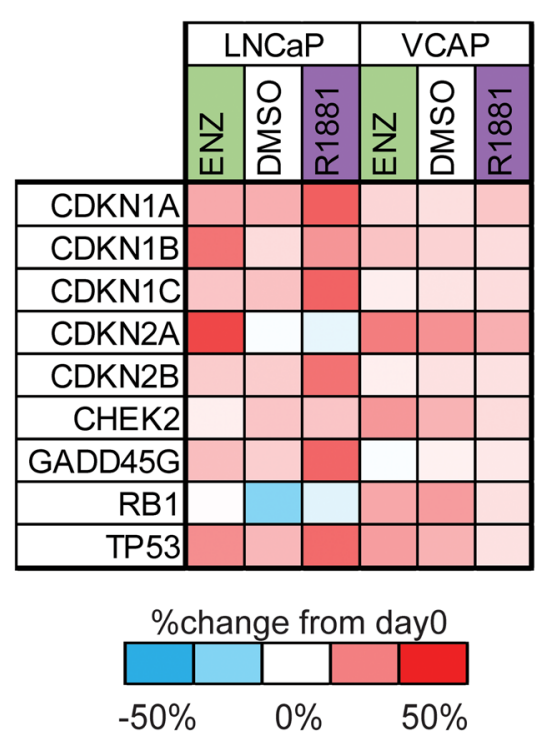

C

$19 \%$

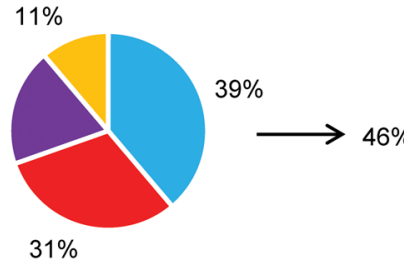

Day 0

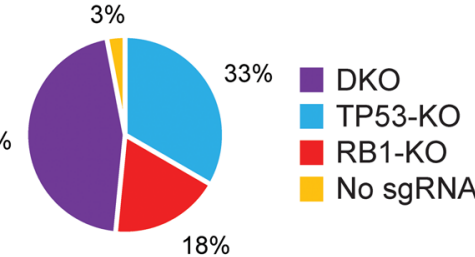

Day 42

Figure 4. Genetic loss-of-function screens identify factors that mediate response to AR-directed therapies. (A) Summary of 4 whole-genome CRISPR/ CAS9 screens. Values (1-4) indicate the number of times the gene appeared in the top 2000 most enriched (+) or depleted (-) sgRNAs by gene. (B) Heatmap of validation study indicating the change in percentage of cells that harbor sgRNAs specific for the indicated gene. (C) Competitive enrichment assay for cells harboring sgRNAs targeting either, both, or neither RB1 and TP53.

Previous studies in PC cells have found E2F1 and AR to coordinately upregulate cell-cycle (44) and DNA-damage response (DDR) genes (45), whereas AR and RB1 coordinately repressed cell-cycle genes (26). To explore the possibility that AR coordinates with RB1 and E2F1 to regulate biphasic genes, we determined the percentage of genes in each of the $4 \mathrm{AR}$ subsets that associated with peaks bound by either AR, RB1, or E2F1, using previously published ChIP-seq data sets for AR (7, 46), E2F1 (44), and RB1 (26). Not surprisingly, binding of AR to genes in the AR-induced gene set was enriched. RB1 and E2F1 were also associated with these genes, but at lower levels. (Figure 6B). Binding of RB1 and E2F1 to biphasic genes was enriched by $17 \%$ and $33 \%$, respectively, whereas AR associated with biphasic genes at a lower percentage in these studies. However, these data do not support or exclude the possibility that AR coordinates with E2F1 and RB1 to regulate biphasic targets. AR typically regulates gene expression via binding to intra- or intergenic enhancers, whereas E2F1/RB1 more directly associates with promoters, making it difficult to unambiguously determine whether an AR-bound enhancer regulates a given biphasic gene. Notably, previous studies have identified AR binding sites associated with the regulation of DDR and cell-cycle genes: AR-induced DDR genes (45) (AR_DDR_UP), AR/RB1-repressed cell-cycle genes (26) (AR_CC_DN), and AR/E2F1-activated cell-cycle genes (44) (AR_CC_UP); there is a high concordance of these gene lists with the biphasic gene category (Figure 6C). The percentage of biphasic genes in these 3 data sets ranged from $53 \%$ to $67 \%$, whereas biphasic genes made up only $4 \%$ of all genes. Interestingly, biphasic genes $M C M 7$ and FANCI were found to be activated by AR in the AR_DDR_UP study and repressed by AR in the AR_CC_DN study, suggesting that the conventional binary classification of 
A

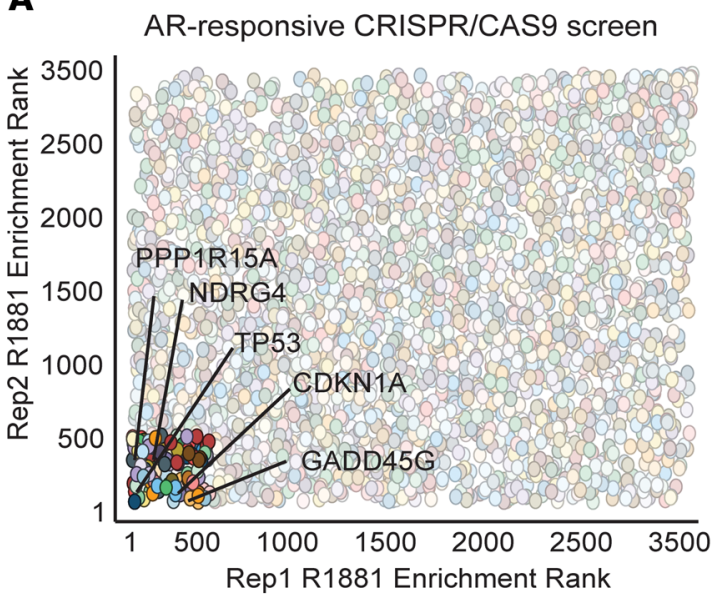

B

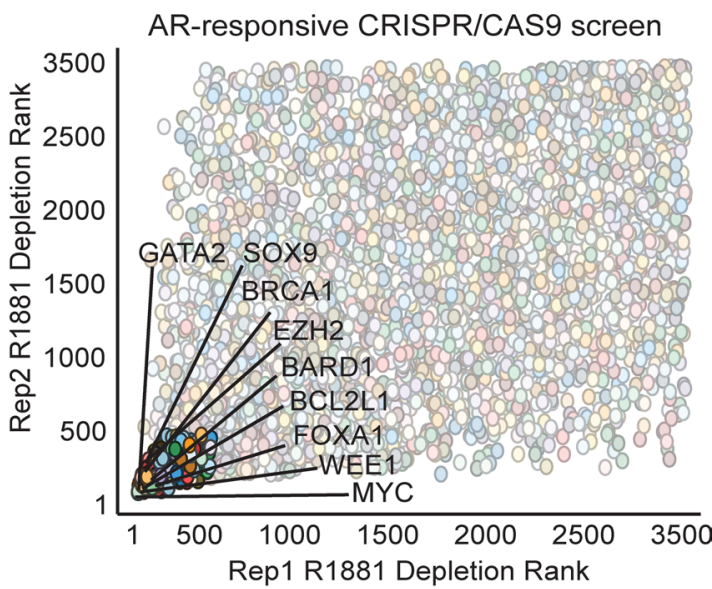

C

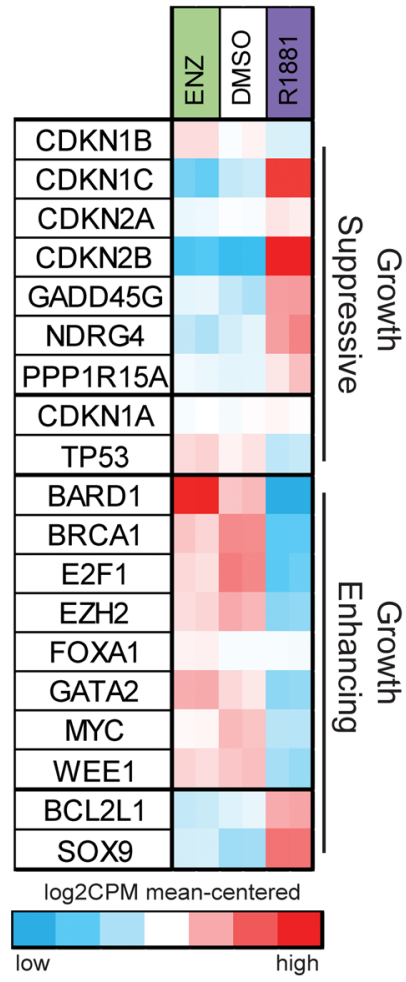

D
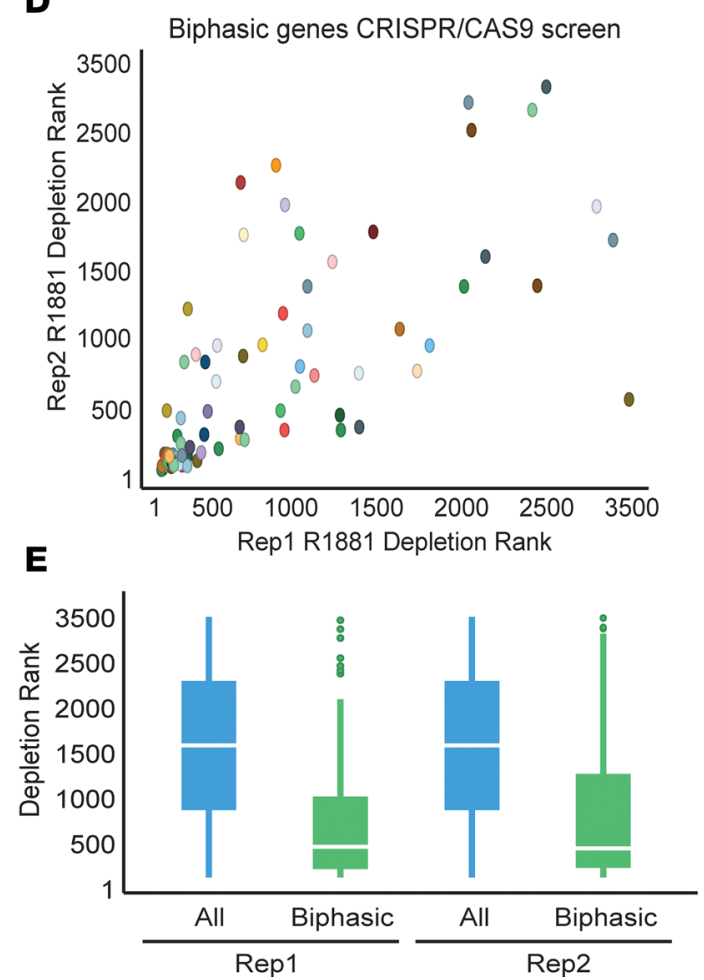

Figure 5. Focused CRISPR/CAS9 screens identify AR-responsive genes that mediate sensitivity to high-dose androgen. (A) Gene enrichment ranks as measured by MACECK analysis of 2 biological replicates of an AR-focused CRISPR/CAS9 screen. Known tumor suppressors enriched in both biological replicates are labeled. (B) Gene depletion ranks of 2 biological replicates. Genes associated with prostate cancer growth and progression are labeled. (C) RNA-seq gene expression heatmaps of mean-centered log2(CPM) values representing genes identified in the whole-genome and focused CRISPR screens. (D) sgRNA depletion rank plot of biphasic genes. (E) Median depletion ranks for biphasic genes (green) compared with all other genes (blue). Bars represent Q1 and Q3 quartile ranges.

AR-induced or AR-repressed does not fully capture the complex regulatory modalities of AR signaling. Together, these data indicate that suppression of biphasically regulated genes may be a fundamental function of AR that is disrupted on oncogenic transformation but may be reestablished by HDA.

Expression profiling of de novo resistance to HDA therapy. We next sought to determine whether cells with de novo resistance to HDA would maintain expression of biphasic genes during HDA. We compared the transcriptional responses of the R1AD1 (47) cell line that was minimally growth repressed by HDA (Figure 1) to the 4 cell lines that were strongly growth repressed by HDA. Compared with the HDA-sensitive cell lines, GSEA analysis of HDA-treated R1AD1 cells revealed a positive enrichment of pathways associated with the biphasic gene set, including cell cycle-related E2F_targets and G2M_checkpoint gene sets, 
A

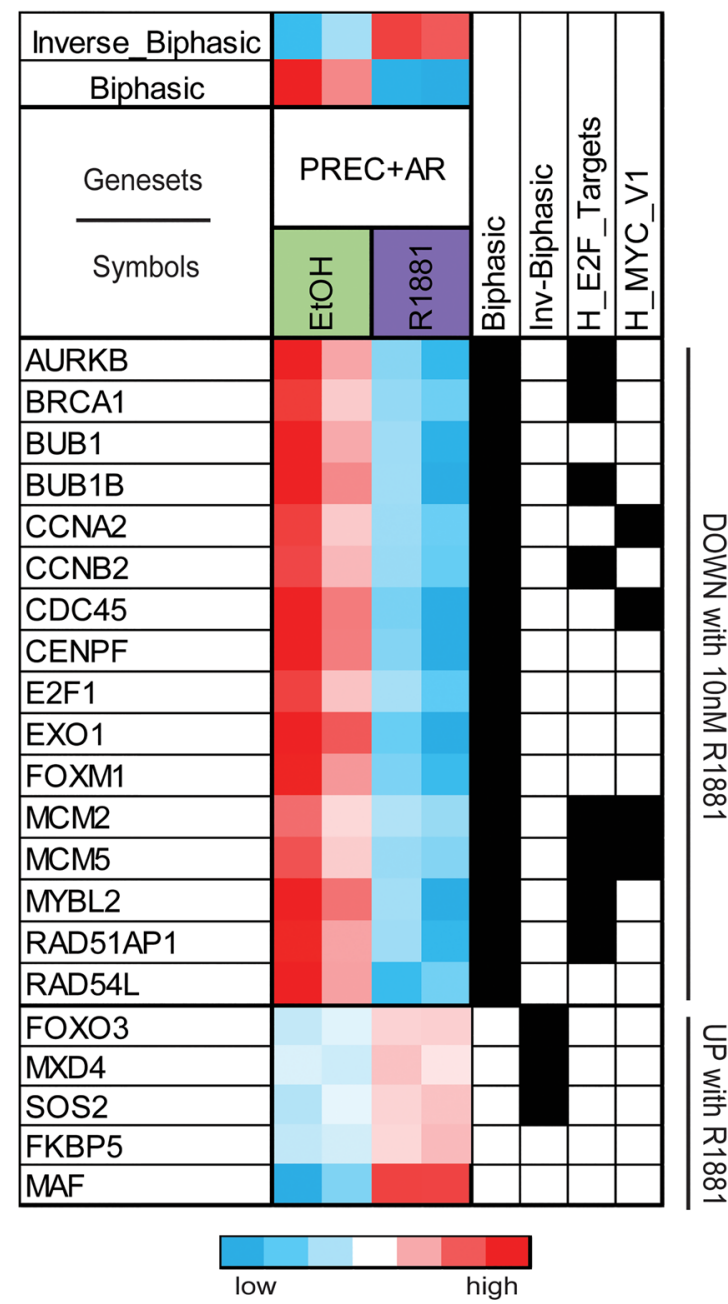

B

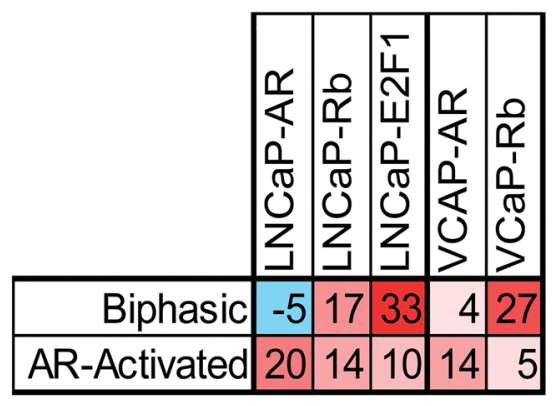

C

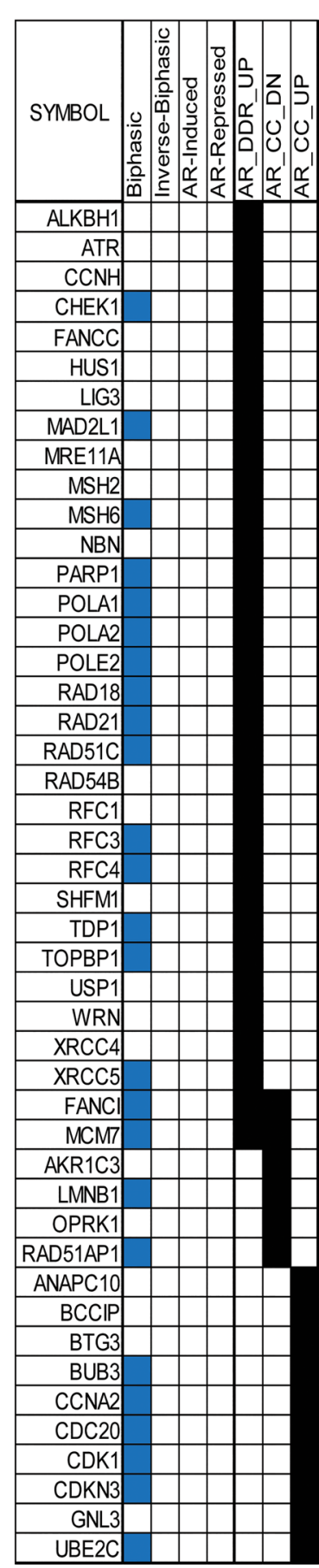

Figure 6. Suppression of biphasic gene expression is an innate function of AR. (A) Heatmaps of custom gene expression signature scores (top) and RNA-seq mean-centered log2(CPM) values (bottom) for the hTERT-immortalized prostate epithelial cell line 957E/hTERT that was engineered to overexpress AR. Cells were treated with either 1 nM R1881 or ethanol. Examples were selected from the top 250 upregulated or downregulated genes. (B) Percentage of genes in each AR gene signature associated with at least 1 ChIP-seq peak for AR, RB1, or E2F1 subtracted by the percentage of genes bound by at least 1 peak in the whole genome. ChIP-seq data sets for both LNCaP and VCAP were compared. (C) Graph of DNA-damage-response (DDR) and cell-cycle (CC) genes previously shown to be bound by $A R$ and are regulated by AR activity. Blue squares indicate the presence of a gene in an AR gene signature for 3 out of 4 HDA-sensitive cell lines. Black bars represent the study that validated the gene as regulated (UP) or downregulated (DN) by androgens. 
as well as plasticity-associated epithelial_mesenchymal_transition (Figure 7A). This model was negatively enriched for gene sets associated with AR-induced genes and luminal prostate differentiation, such as androgen_reponse, oxidative_phosphorylation, adipogenesis, cholesterol_homeostasis, protein_secretion, and fatty_acid_metabolism (Figure 7A).

Accordingly, numerous oncogenes and drivers of cell cycle, including 29 biphasic genes, were within the top 250 genes expressed most highly expressed in R1881-treated R1AD1 cells (Figure 7B and Supplemental Table 6). Biphasic genes, including several E2F targets, were only weakly repressed by ENZ and R1881. In addition, oncogenes that drive proliferation, such as $M Y C L, C D K 6$, and CCND1, were highly expressed (Figure 7B). This attenuated repression of biphasic genes by R1881 can be seen in FOXM1 and CCNA2 (Figure 7, C and D).

Consistent with the GSEA data showing negative enrichment of the AR-induced gene set in R1AD1 cells, the 250 most downregulated genes with R1881 between R1AD1 and HDA-sensitive lines included 82 AR-induced genes, such as TMPRSS2, PEMPA1, and AMACR (Figure 7E and Supplemental Table 7). Several important AR-coregulators were also expressed at very low levels in R1AD1 cells, including HOXB13, GATA2, SOX9, and FOXA1 (Figure 7E). Interestingly, MYC was significantly suppressed under R1881 conditions in R1AD1 cells (Figure 7F), suggesting HDA resistance in R1AD1 cells was not dependent on maintaining MYC expression. Taken together, these data indicate that induction of cellular differentiation and inhibition of mitogenic pathways are partially uncoupled from the control of AR in R1AD1 cells, resulting in resistance to AR-directed therapies.

Predicting therapy response using biphasic and AR gene signatures. Gene set variation analysis (GSVA) (48) was then used to compare the 4 gene set signatures identified in this study to previously published gene sets defining AR regulation (ARG.10), cell cycle/proliferation (CCP.31), epithelial-to-mesenchymal transformation (EMT.12), and the neuroendocrine phenotype (NE.10) (ref. 49 and Supplemental Table 8). HDA-sensitive cell models showed strong upregulation of AR-induced and ARG.10 genes and strong repression of the AR-repressed, biphasic, and CCP.31 gene sets in response to HDA, whereas none of these gene sets were strongly affected by HDA in R1AD1 (Figure 8A). R1AD1 also had constitutively low AR-repressed and inverse-biphasic gene signatures. Neither the epithelial-to-mesenchymal transition (EMT.12) or the neuroendocrine signatures (NE.10) distinguished R1AD1 from the other cell lines (Figure 8A). However, the HDA-resistant model R1AD1 could be clearly distinguished from the HDA-sensitive models by having a high biphasic/proliferative but a low AR-induced gene signature under all treatment conditions, including vehicle (Figure 8A).

Analogous tumor samples with a high biphasic/proliferative but a low AR-induced gene signature can be identified in the RNA-seq profiles of 4212 CRPC metastases in the PCF/Stand-Up-To-Cancer cohort (Figure 8B). Similar to R1AD1, tumors that have high biphasic/proliferation signatures but low AR-induced signatures (quadrant 1, Q1) have uncoupled AR-signaling from the control of proliferation and would likely be poor candidates for HDA-based therapies (Figure 8B). Tumors that have high biphasic/ proliferation and high AR-induced signatures (Q2) are more likely to have achieved castration resistance through reactivation of $\mathrm{AR}$ signaling and may be good candidates for HDA therapy (Figure 8B). Interestingly, several of the candidate responders in Q2 also had high neuroendocrine signature scores (NE.10), analogous to $\mathrm{VCaP}$ cells, raising the possibility that some $\mathrm{NE}^{+} / \mathrm{AR}^{+}$or amphicrine $\mathrm{PC}$ subtypes may also benefit from HDA-based therapies.

To determine whether AR signatures can be used as an early indicator of therapy response, we applied them to RNA-seq data from a recent study of the LuCaP CRPC PDX models treated with HDA (50). RNA from responder tumor lines (LuCaP96CR and $\mathrm{LuCaP35CR}$ ) and one nonresponder line (LuCaP77CR) were harvested at an early time point, 5 days into treatment. Although all lines had significant reductions $(P$ $<0.05$ ) in biphasic gene signature scores in response to testosterone, expression of biphasic genes remained high in the nonresponder line (Figure 8C). In contrast, all PDX lines had similar increases in expression of the inverse-biphasic (Figure 8D) and AR-induced gene sets (Figure 8E) and decreases in the AR-repressed gene signature (Figure $8 \mathrm{~F}$ ).

\section{Discussion}

Patients with PC display a range of radiographic and prostate-specific antigen (PSA) responses to HDAbased therapies (11) but the factors that mediate response or resistance to AR-mediated growth suppression are not well understood. Studies of AR signaling commonly use CSS-media with or without high doses of 
A

R1AD1 vs HDA-sensitive
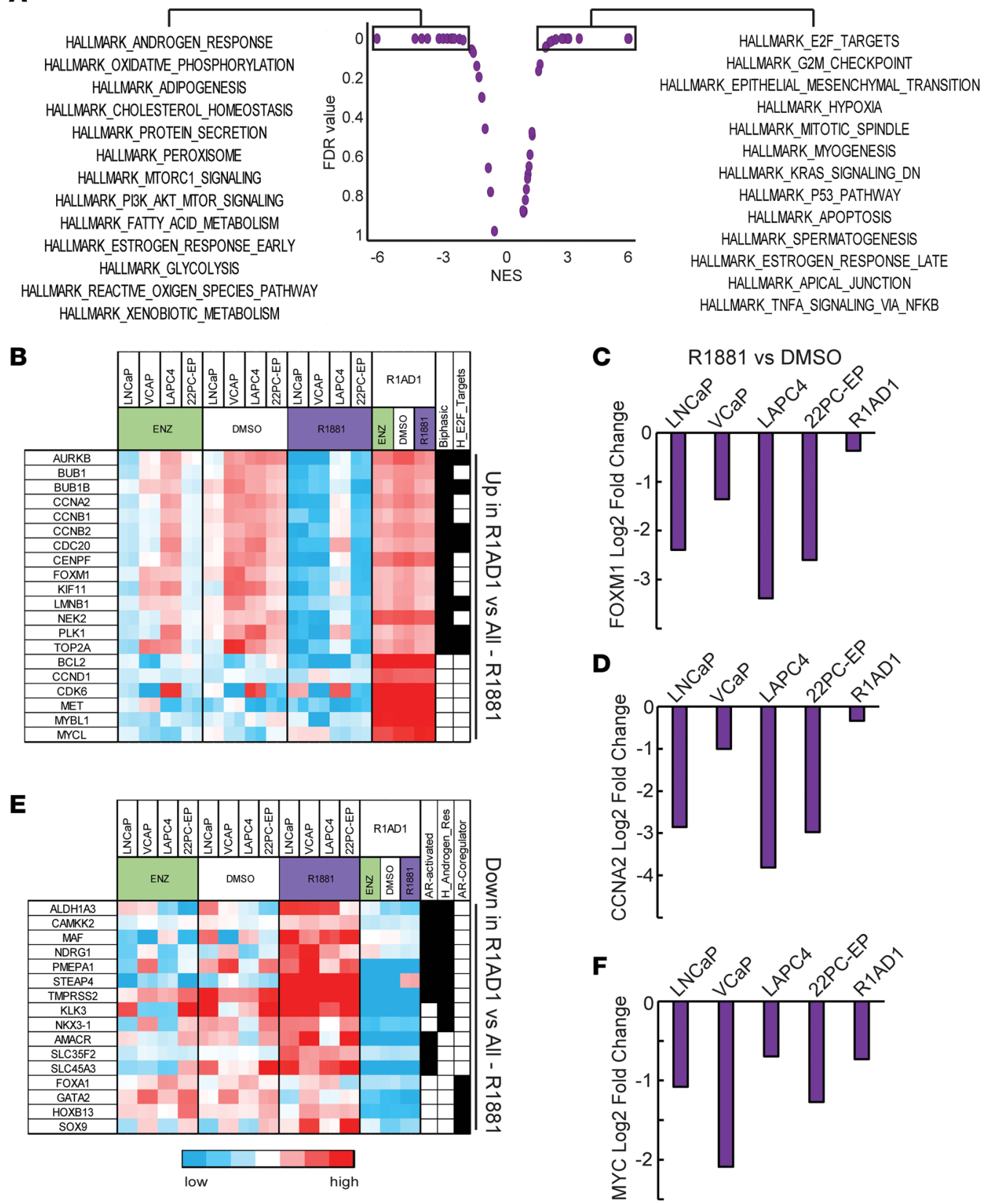

Figure 7. De novo resistance to high-dose androgen is associated with loss of differentiation and gain of a mitotic gene expression profile. (A) Gene set enrichment analysis (GSEA) plots of normalized-enrichment scores (NES) and FDR values for differentially expressed genes between R1AD1 and the 4 HDA-sensitive cell lines when treated with 10 nM R1881 (FDR < 0.05). (B) Heatmap of RNA-seq Log2(CPM) values, mean-centered across all cell lines, of a selection of the 250 most upregulated genes in R1AD1 cells treated compared with LNCaP, VCAP, LAPC4, and 22PC-EP when treated with $10 \mathrm{nM} \mathrm{R} 1881$ (bottom). Black squares indicate positive status as a biphasic gene or Hallmark_E2F_target gene. Log2(fold change) in gene expression from vehicle control when treated with $10 \mathrm{nM}$ R1881 for (C) FOXM1 and (D) CCNA2. (E) Heatmaps of RNA-seq mean-centered Log2(CPM) values of a subset of the 250 most downregulated genes in R1AD1 compared with LNCaP, VCAP, LAPC4, and 22PC-EP when treated with $10 \mathrm{nM}$ R1881 (bottom). Black squares indicate positive status as an AR-induced, Hallmark_Androgen_Response, or AR-coregulator gene. (F) Log2(fold change) in MYC expression from vehicle control when treated with 10 nM R1881.

androgen. As a result, genes are either classified as AR-activated or AR-suppressed. However, by tracking how individual genes changed through a phenotypically defined AR activity spectrum consisting of AR-inhibited growth repression, normal proliferation, and AR-hyperactive growth repression, AR-signaling modalities can be subset into 4 categories: (a) AR-induced genes representing biosynthetic processes; (b) 
A
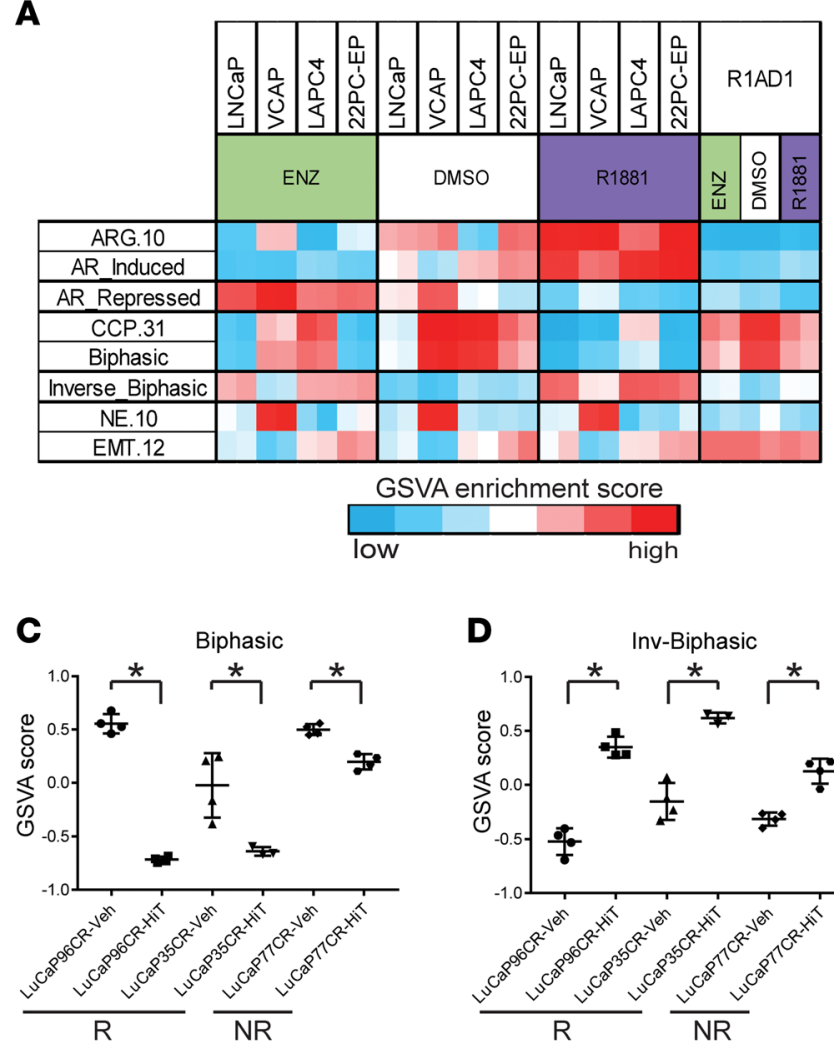

D

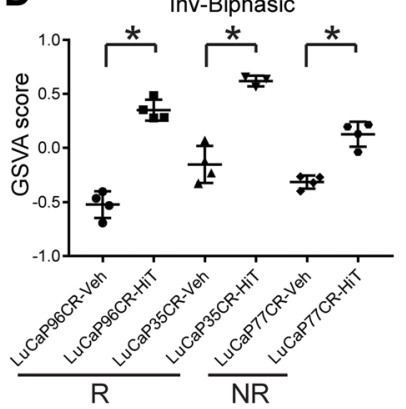

G

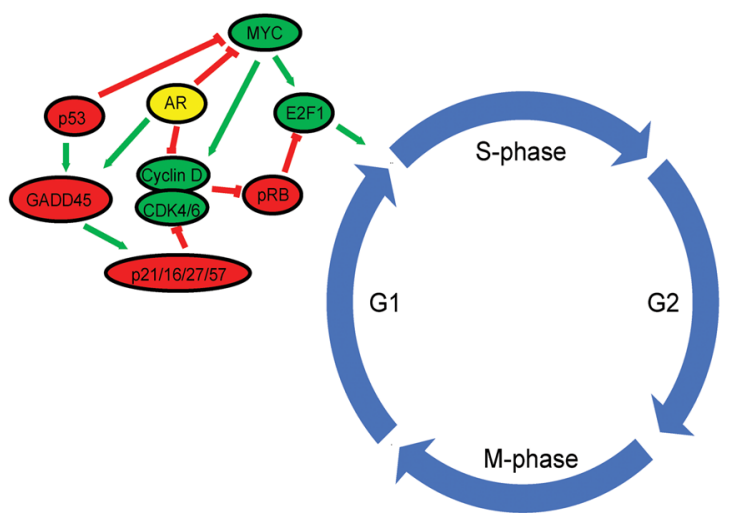

B

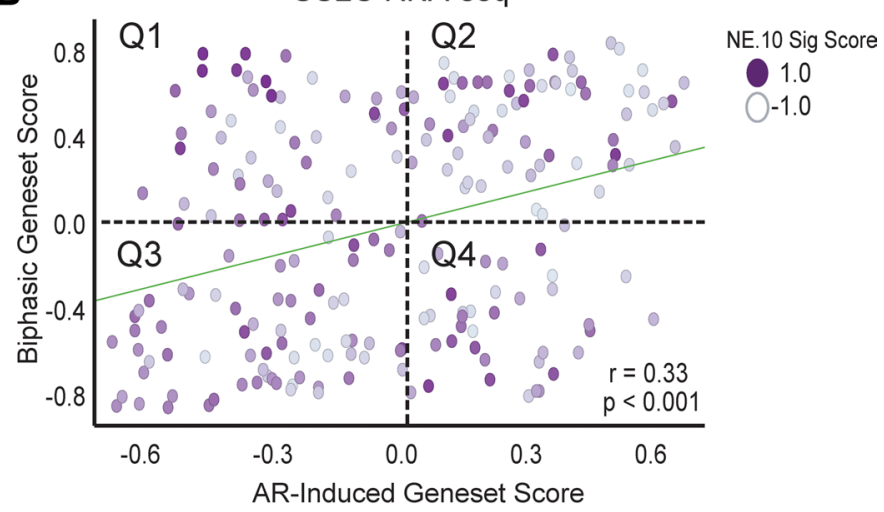

E

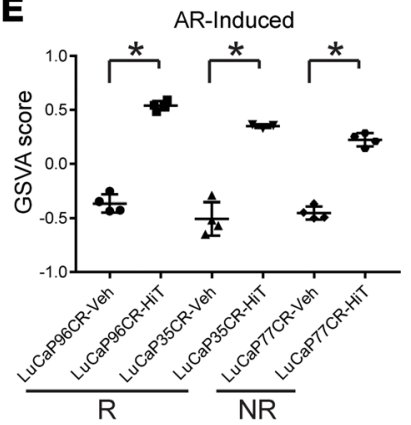

$\mathbf{F}$

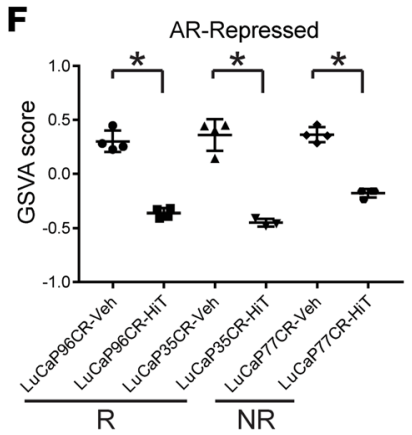

H

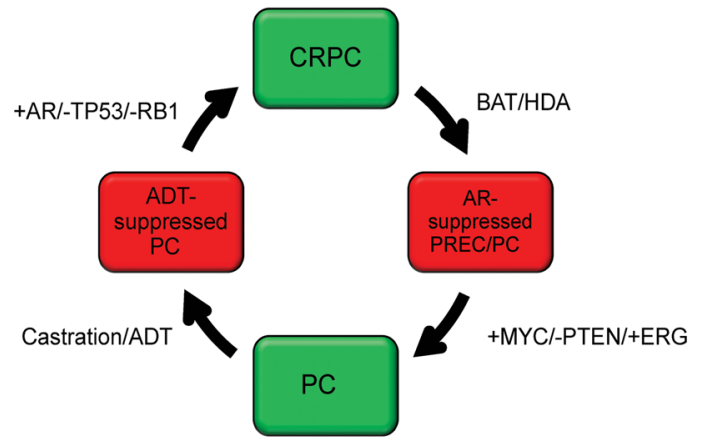

Figure 8. AR signature scores identify potential responders in clinical castration-resistant prostate cancer samples. (A) Heatmaps of gene set variation analysis (GSVA) signature scores comparing previously published gene signatures with gene signatures derived in the current study. (B) Correlation of biphasic and AR-induced signature scores in $n=212$ CRPC tumor specimens, colored by neuroendocrine (NE.10) signature score values. Quadrants Q1-Q4 are delineated by hatched lines. Signature scores are indicated for vehicle-treated (veh) or high-dose testosterone-treated (HiT) responder (R) or nonresponder (NR) PDX lines for (C) biphasic, (D) inverse-biphasic, (E) AR-induced, and (F) AR-repressed gene sets. (G) Diagram of an interactive web of factors that mediate sensitivity (red) and resistance (green) to AR-directed therapies. (H) The relationship between AR signaling and prostate cancer throughout disease progression is diagrammed.

AR-repressed genes involved in regulation of protein translation; (c) inverse-biphasic genes - upregulated in cell cycle-suppressed states and including important stress-response genes in the FOXO family; and (d) biphasic genes representing critical drivers of proliferation.

Although numerous mechanisms have been proposed to explain the growth suppressive effect of HDA, they primarily conform to one of two models. In one model, AR activity suppresses an oncogene or upregulates a tumor suppressor, either directly or in conjunction with a metabolic stress response to ROS or ER stress $(13-17,26,51)$. In another model, acute activation of AR by HDA, when preceded by several days of androgen withdrawal, induces DNA damage leading to a stress response and cell-cycle suppression $(6,18-20)$. In the former model, no single factor regulated by AR has been identified that can completely account for the 
growth-suppressed phenotype. In the latter model, the connection between the acute and transient DNA-damage-signaling foci observed after acute AR stimulation and the subsequent phenotype of growth suppression after culturing cells with androgens continuously for several days has not been established (9).

Our data suggest that sensitivity or resistance to HDA and ADT is multifactorial, and a continuum of phenotypes likely exist. Genetic screening established that numerous genes mediating co-resistance to ADT and HDA converged to regulate cell-cycle and biphasic gene expression. Integrating RNA-seq data sets with a custom, AR-focused, CRISPR/CAS9 screen revealed that HDA-mediated AR activity suppressed growth through the upregulation of tumor suppressors, such as $C D K N$-family genes, $P P P 1 R 15 A$, and GADD45G, as well as through the suppression of factors that drive PC growth, such as $E Z H 2, M Y C$, and $E 2 F 1$. These data also support the conclusion that de novo resistance to HDA therapy may be co-acquired with ADT resistance through the uncoupling of AR from control of the cell cycle, potentially explaining why not all patients with CRPC are sensitive to HDA therapy. Another means by which cells could evade suppressive AR functions is through changes to cellular plasticity. In this regard, the ORF screen data suggest that overexpression of reprogramming factors LIN28A and SOX2 (52) many enhance resistance. In addition, the HDA-resistant line R1AD1 overexpressed oncogenes not seen in the HDA-sensitive lines, such as MYCL, MET, and BCL2.

Homologous recombination deficiency (HRD) has been suggested to indicate sensitivity to HDA (12); however, the genomic changes in HRD-related genes in our cell models precluded a meaningful analysis. For example, although the responder cell line $\mathrm{LNCaP}$ has a heterozygous CHEK2 mutation, the other responder cell lines, including our nontransformed epithelial cell line, are not known to have HRD mutations (53). In contrast, the nonresponder R1AD1 is a subclone of the CWR-R1 tumor that may have a defect in the HR gene MRE11 (53). Moreover, whereas a responder PDX model, LuCaP $96 \mathrm{CR}$, has BRCA2 loss, the other responder, LuCaP $35 \mathrm{CR}$, is HRD-proficient (50). Our data do not support or refute the hypothesis that sensitivity to HDA therapy is related to DNA damage; however, the data demonstrate that factors other than DNA repair are involved in the response to HDA.

$\mathrm{AR}$ is a master regulator of cell homeostasis controlling the maintenance of prostatic cellular identity and metabolism. Accordingly, AR signaling is likely to be integrated into negative-feedback mechanisms that dynamically regulate cell physiology when hormone signaling is perturbed. Overall, these experiments support a model wherein AR signaling influences a network of tumor suppressors and oncogenes that regulate cell-cycle progression in normal and transformed prostatic tissues (Figure 8G). In this model, partial uncoupling of AR from control of cell cycle through gain of oncogenes, such as MYC and TMPRSS2-ERG, or loss of tumor suppressors, such as PTEN, leads to oncogenic AR activities and PC. CRPC results from further dysregulation of growth through loss of tumor suppressors or activation of promiscuous and/or ligand independent AR signaling through AR mutation or amplification. In the latter case, growth suppression and re-differentiation may be induced in CRPC through hyperstimulation of AR (Figure 8H).

CRPC samples with high proliferation scores have variable AR activity scores. This diversity may be a manifestation of the varied and complex paths taken to castration resistance or androgen independence. Tumors able to uncouple cell cycle from AR control through gain of oncogenes, loss of tumor suppressors, or acquisition of a plastic phenotype are likely to be highly resistant to any AR-directed therapies and will be manifested by high biphasic and low AR-induced signature scores. Alternatively, cells that gain castration resistance mainly through aberrant activation of AR signaling, achieved by AR mutation, $A R$ amplification, or transcriptional induction of active AR variants, are likely to be sensitive to HDA. Cancers with persistent AR signaling in the context of AR mutations that render them resistant to antiandrogens may be excellent candidates for HDA therapy. Tumors with AR variants generated by structural gene rearrangements that eliminate the ligand binding domain (47) are unlikely to be sensitive to HDA. However, these represent a minority of tumors, and expression of the more commonly encountered AR variants generated by alternative splicing does not preclude sensitivity to HDA because repletion of androgen is associated with suppression of AR variant expression (54).

Suppression of PC growth by HDA is a new therapeutic approach with the potential to prolong the efficacy of AR-directed therapies while improving quality of life. The need to identify cancers with de novo resistance either prospectively or soon after treatment has begun is critical so that alternative therapies can be administered. Given the diversity of AR signaling and the multitude of genetic factors that regulate the cell cycle, gene signatures may have a superior ability to identify responders compared with monogenic biomarkers such as PSA or MYC. Evaluation of the LuCaP PDX models suggests that solid or liquid tumor biopsies obtained early (within 1 week) after initiation of HDA that show repression of the biphasic gene signature derived from this investigation may identify men with tumors amenable to HDA-based therapies 
and serve as an early measure of therapy response. To implement these signatures into clinical practice, gene expression profiles of CRPC patient tumors or circulating tumor cells prior to and during HDA therapy should be correlated with outcomes to define the optimal criteria for their use.

\section{Methods}

Dose-response assays. Cells lines were obtained from ATCC and used within 20 passages or provided by other laboratories (see Acknowledgments). Dose response assays were carried out in 96-well plates $(n=4)$ in 100 $\mu \mathrm{L} /$ well of $10 \%$ FBS or $10 \%$ CSS supplemented phenol-free RPMI-1640 (Thermo Fisher Scientific, 11835 030) for LNCaPs, R1AD1, 22Rv1 and 22PC-EP or DMEM/F12 (Thermo Fisher Scientific, 11039021). Cells were cultured for 5 days, then harvested with CellTiter-Glo 2.0 (Promega, G9241) and measured on a Synergy plate reader (BioTek). 22PC-EP were a gift from the laboratory of Charles Sawyers (Memorial Sloan Kettering Cancer Center, New York, New York, USA).

Competitive enrichment assays. To create the ORF vectors for the competition assay, ORFs were amplified from an arrayed ORF library (55), TOPO-TA cloned into PCR8/GW (Thermo Fisher Scientific, K250020), then gateway cloned into the FU-CGW lentiviral vector (a gift from the laboratory of Owen Witte, UCLA, Los Angeles, California, USA) using LR clonase (Thermo Fisher Scientific, 11791100). Transduced cells were mixed in a 1:1 ratio with the FU-CRW control vector and cultured in normal growth conditions with DMSO vehicle, $10 \mathrm{nM}$ R1881, or $10 \mu \mathrm{M}$ enzalutamide (Selleckchem, S1250). A day 0 time point was analyzed by flow cytometry. The remaining cells were cultured for 25 (LNCaP) or 30 (VCAP) days and then analyzed by flow cytometry. Dual TP53 and RB1 competition assays were cultured in R1881 for 42 days.

CRISPR/CAS9 competition assays were performed similarly using the pZHB-Z:U6_EFS-GFP (or mCherry)-Puro sgRNA vector (a gift from the laboratory of Patrick Paddison, Fred Hutchinson Cancer Research Center, Seattle, Washington, USA), which coexpressed either RFP or GFP with the nontargeting control and test sgRNAs, respectively. Test sgRNAs were designed using Broad design tool (portals.broadinstitute.org/gpp/public/analysis-tools/sgrna-design) and subcloned using ESP3I sites and overlapping annealed oligonucleotides (www.idtdna.com).

$R N A$-seq analysis. Cells $\left(1.5 \times 10^{6}\right)$ were plated in 6-well plates in normal growth media; 24 hours later $10 \mu \mathrm{M}$ ENZ (SelleckChem, S1250) or $10 \mathrm{nM}$ R1881 (Sigma-Aldrich, R0908) was added and cultured for 48 hours, at which point the cells were harvested using an RNeasy Kit (Qiagen, 74104). PREC lines were treated with $1 \mu \mathrm{g} / \mathrm{mL}$ doxycycline (Sigma-Aldrich, D9891) and $1 \mathrm{nM} \mathrm{R} 1881$ and harvested after 24 hours. Libraries were prepared, sequenced, and aligned as previously described (49). Differentially regulated genes were analyzed using EdgeR (56). GSEA was performed as described previously (28) using preranked gene lists with classic weighting. GSVA was performed using the GSVA R-package (48). PANTHER GO overrepresentation tests were performed using the online tool (www.pantherdb.org) as previously described (30).

CRISPR/ORF screens. The human GeCKOv2 CRISPR KO pooled library was obtained from Feng Zhang (Addgene 1000000048, 1000000049). LNCaP cells were transduced with the pooled sgRNA libraries and cultured for 25 days with $10 \mu \mathrm{M}$ ENZ, $10 \mathrm{nM}$ R1881, or DMSO vehicle control. Cells $\left(4 \times 10^{7}\right)$ were used per group for a 100- to 200-fold coverage of the library. DNA was harvested using the Blood \& Cell Culture DNA Midi Kit (Qiagen, 13343). Barcode amplification was performed with 2 rounds of amplification using Phusion-HF polymerase (Thermo Fisher Scientific, F530L), each 14 cycles, using primers in Supplemental Table 2 and sequenced as previously described (36). Hits were identified using MAGECK analysis (37, 38).

The custom AR-regulated CRISPR screen was designed using optimized sgRNAs assembled from several studies (refs. 57-59 and Supplemental Table 3). Oligonucleotides were ordered from custom array (www.customarrayinc.com), PCR amplified, and subcloned into a custom vector pZHB-Z:U6_EFS-GFPPuro, a gift from the laboratory of Patrick Paddison, using Esp3I enzyme sites (Thermo Fisher Scientific, ER0451) and T4 ligase (NEB, M0202S). The screen was performed and sequenced similarly to the whole genome, except that $2 \times 10^{7}$ cells were used to achieve $500 \times$ library coverage.

The TRC ORF screen, a gift from the laboratory of Patrick Paddison, was performed similarly to the custom CRISPR screen with 2 rounds of barcode amplification using primers in Supplemental Table 2.

ChIP-seq peak annotation. ChIP-seq data sets were downloaded from the GEO database (www.ncbi.nlm. nih.gov/geo) as .bed files and converted to HG38 using the liftover tool (https://genome.ucsc.edu/cgi-bin/ hgLiftOver). Peaks were annotated using the PAVIS tool (https://manticore.niehs.nih.gov/pavis2/) using default settings. The following data sets were used: AR in VCAP, GSM801011; AR in LNCAP, GSM980662; Rb VCAP, GSM1974981; Rb LNCAP, GSM1974982; Rb VCaP, GSM1974981; E2F1 LNCaP, GSM2492421. 
Statistics. Significance was defined by an FDR value of less than 0.05 as determined by EdgeR for differentially expressed RNA-seq values; PANTHER GO and GSEA for pathway and gene set analysis; MACS2 for ChIP-seq peak calling; and PAVIS for gene annotation.

Study approval. No human or animal subjects were used in this study.

\section{Author contributions}

MDN, AC, OM, and JML carried out in vitro experiments and RNA-seq analysis. IC and AK performed RNA-seq. PJP and DAK advised on the design and analysis of the sgRNA screen. MDN, SRP, PSN, and EAM designed the study and edited the manuscript. MDN and EAM wrote the manuscript.

\section{Acknowledgments}

We would like to thank John Isaacs John Isaacs (Johns Hopkins Hospital, Baltimore, Maryland, USA) for providing PREC/AR cells and Charles Sawyers for providing 22PC-EP cells. This study was supported by the following grants: W81XWH-15-1-0319, W81XWH-16-1-0206, P01 CA163227, P50 CA097186, P30 CA015704, and R21 CA230138.

Address correspondence to: Elahe A. Mostaghel, Geriatric Research, Education and Clinical Center S-182, VA Puget Sound Health Care System, 1660 South Columbian Way, Seattle, Washington 98108-1597, USA. Phone: 206.277.1657; Email: emostagh@fredhutch.org.

1. Cary KC, Singla N, Cowan JE, Carroll PR, Cooperberg MR. Impact of androgen deprivation therapy on mental and emotional well-being in men with prostate cancer: analysis from the CaPSURE ${ }^{\mathrm{TM}}$ registry. J Urol. 2014;191(4):964-970.

2. de Bono JS, et al. Abiraterone and increased survival in metastatic prostate cancer. N Engl J Med. 2011;364(21):1995-2005

3. Scher HI, et al. Increased survival with enzalutamide in prostate cancer after chemotherapy. N Engl J Med. 2012;367(13):1187-1197.

4. Watson PA, Arora VK, Sawyers CL. Emerging mechanisms of resistance to androgen receptor inhibitors in prostate cancer. Nat Rev Cancer. 2015;15(12):701-711.

5. Denmeade SR, Isaacs JT. Bipolar androgen therapy: the rationale for rapid cycling of supraphysiologic androgen/ablation in men with castration resistant prostate cancer. Prostate. 2010;70(14):1600-1607.

6. Hedayati M, et al. Androgen deprivation followed by acute androgen stimulation selectively sensitizes AR-positive prostate cancer cells to ionizing radiation. Clin Cancer Res. 2016;22(13):3310-3319.

7. Cai C, et al. Androgen receptor gene expression in prostate cancer is directly suppressed by the androgen receptor through recruitment of lysine-specific demethylase 1. Cancer Cell. 2011;20(4):457-471.

8. Isaacs JT, et al. Adaptive auto-regulation of androgen receptor provides a paradigm shifting rationale for bipolar androgen therapy (BAT) for castrate resistant human prostate cancer. Prostate. 2012;72(14):1491-1505.

9. Schweizer MT, et al. Effect of bipolar androgen therapy for asymptomatic men with castration-resistant prostate cancer: results from a pilot clinical study. Sci Transl Med. 2015;7(269):269ra2.

10. Schweizer MT, et al. Bipolar androgen therapy for men with androgen ablation naïve prostate cancer: results from the phase II BATMAN Study. Prostate. 2016;76(13):1218-1226.

11. Teply BA, et al. Bipolar androgen therapy in men with metastatic castration-resistant prostate cancer after progression on enzalutamide: an open-label, phase 2, multicohort study. Lancet Oncol. 2018;19(1):76-86.

12. Teply BA, Kachhap S, Eisenberger MA, Denmeade SR. Extreme response to high-dose testosterone in BRCA2- and ATM-mutated prostate cancer. Eur Urol. 2017;71(3):499.

13. Kim YC, Chen C, Bolton EC. Androgen receptor-mediated growth suppression of HPr-1AR and PC3-Lenti-AR prostate epithelial cells. PLoS One. 2015;10(9):e0138286.

14. Daniels G, Jha R, Shen Y, Logan SK, Lee P. Androgen receptor coactivators that inhibit prostate cancer growth. Am J Clin Exp Urol. 2014;2(1):62-70.

15. Wen S, Niu Y, Lee SO, Chang C. Androgen receptor (AR) positive vs negative roles in prostate cancer cell deaths including apoptosis, anoikis, entosis, necrosis and autophagic cell death. Cancer Treat Rev. 2014;40(1):31-40.

16. Vander Griend DJ, Litvinov IV, Isaacs JT. Conversion of androgen receptor signaling from a growth suppressor in normal prostate epithelial cells to an oncogene in prostate cancer cells involves a gain of function in c-Myc regulation. Int J Biol Sci. 2014;10(6):627-642.

17. Zhang L, et al. Androgen suppresses protein kinase D1 expression through fibroblast growth factor receptor substrate 2 in prostate cancer cells. Oncotarget. 2017;8(8):12800-12811.

18. Haffner MC, et al. Androgen-induced TOP2B-mediated double-strand breaks and prostate cancer gene rearrangements. Nat Genet. 2010;42(8):668-675.

19. Roediger J, et al. Supraphysiological androgen levels induce cellular senescence in human prostate cancer cells through the SrcAkt pathway. Mol Cancer. 2014;13:214.

20. Pinthus $\mathrm{JH}$, et al. Androgen induces adaptation to oxidative stress in prostate cancer: implications for treatment with radiation therapy. Neoplasia. 2007;9(1):68-80.

21. Bui AT, Huang ME, Havard M, Laurent-Tchenio F, Dautry F, Tchenio T. Transient exposure to androgens induces a remarkable self-sustained quiescent state in dispersed prostate cancer cells. Cell Cycle. 2017;16(9):879-893. 
22. Mirochnik Y, et al. Androgen receptor drives cellular senescence. PLoS One. 2012;7(3):e31052.

23. Litvinov IV, et al. Androgen receptor as a licensing factor for DNA replication in androgen-sensitive prostate cancer cells. Proc Natl Acad Sci U S A. 2006;103(41):15085-15090.

24. Sheng X, et al. Divergent androgen regulation of unfolded protein response pathways drives prostate cancer. $E M B O M o l ~ M e d$. 2015;7(6):788-801.

25. Segawa T, et al. Androgen-induced expression of endoplasmic reticulum (ER) stress response genes in prostate cancer cells. Oncogene. 2002;21(57):8749-8758.

26. Gao S, et al. Androgen receptor tumor suppressor function is mediated by recruitment of retinoblastoma protein. Cell Rep. 2016;17(4):966-976.

27. Tu C, et al. Proteomic analysis of charcoal-stripped fetal bovine serum reveals changes in the insulin-like growth factor signaling pathway. J Proteome Res. 2018;17(9):2963-2977.

28. Sedelaar JP, Isaacs JT. Tissue culture media supplemented with $10 \%$ fetal calf serum contains a castrate level of testosterone. Prostate. 2009;69(16):1724-1729.

29. Mootha VK, et al. PGC-1 $\alpha$-responsive genes involved in oxidative phosphorylation are coordinately downregulated in human diabetes. Nat Genet. 2003;34(3):267-273.

30. Mi H, Muruganujan A, Casagrande JT, Thomas PD. Large-scale gene function analysis with the PANTHER classification system. Nat Protoc. 2013;8(8):1551-1566.

31. Huang WC, Li X, Liu J, Lin J, Chung LW. Activation of androgen receptor, lipogenesis, and oxidative stress converged by SREBP-1 is responsible for regulating growth and progression of prostate cancer cells. Mol Cancer Res. 2012;10(1):133-142.

32. Audet-Walsh E, et al. SREBF1 activity is regulated by an AR/mTOR nuclear axis in prostate cancer. Mol Cancer Res. 2018;16(9):1396-1405.

33. Rodriguez-Bravo V, Carceles-Cordon M, Hoshida Y, Cordon-Cardo C, Galsky MD, Domingo-Domenech J. The role of GATA2 in lethal prostate cancer aggressiveness. Nat Rev Urol. 2017;14(1):38-48.

34. He B, et al. GATA2 facilitates steroid receptor coactivator recruitment to the androgen receptor complex. Proc Natl Acad Sci U S A 2014;111(51):18261-18266.

35. Robinson D, et al. Integrative clinical genomics of advanced prostate cancer. Cell. 2015;162(2):454.

36. Sanjana NE, Shalem O, Zhang F. Improved vectors and genome-wide libraries for CRISPR screening. Nat Methods. 2014;11(8):783-784.

37. Li W, et al. MAGeCK enables robust identification of essential genes from genome-scale CRISPR/Cas9 knockout screens. Genome Biol. 2014;15(12):554.

38. Li W, et al. Quality control, modeling, and visualization of CRISPR screens with MAGeCK-VISPR. Genome Biol. 2015 ;16:281.

39. Tiwari N, et al. Sox 4 is a master regulator of epithelial-mesenchymal transition by controlling Ezh2 expression and epigenetic reprogramming. Cancer Cell. 2013;23(6):768-783.

40. Bilir B, et al. SOX4 is essential for prostate tumorigenesis initiated by PTEN ablation. Cancer Res. 2016;76(5):1112-1121.

41. Feng FY, et al. MDM2 inhibition sensitizes prostate cancer cells to androgen ablation and radiotherapy in a p53-dependent manner. Neoplasia. 2016;18(4):213-222.

42. Williams BO, Remington L, Albert DM, Mukai S, Bronson RT, Jacks T. Cooperative tumorigenic effects of germline mutations in $\mathrm{Rb}$ and p53. Nat Genet. 1994;7(4):480-484.

43. Antony L, van der Schoor F, Dalrymple SL, Isaacs JT. Androgen receptor (AR) suppresses normal human prostate epithelial cell proliferation via AR/ $\beta$-catenin/TCF-4 complex inhibition of c-MYC transcription. Prostate. 2014;74(11):1118-1131.

44. McNair C, et al. Differential impact of RB status on E2F1 reprogramming in human cancer. J Clin Invest. 2018;128(1):341-358.

45. Polkinghorn WR, et al. Androgen receptor signaling regulates DNA repair in prostate cancers. Cancer Discov. 2013;3(11):1245-1253.

46. Sahu B, et al. FoxA1 specifies unique androgen and glucocorticoid receptor binding events in prostate cancer cells. Cancer Res. 2013;73(5):1570-1580.

47. Nyquist $\mathrm{MD}$, et al. TALEN-engineered AR gene rearrangements reveal endocrine uncoupling of androgen receptor in prostate cancer. Proc Natl Acad Sci U S A. 2013;110(43):17492-17497.

48. Hänzelmann S, Castelo R, Guinney J. GSVA: gene set variation analysis for microarray and RNA-seq data. BMC Bioinformatics. 2013;14:7.

49. Bluemn EG, et al. Androgen receptor pathway-independent prostate cancer is sustained through FGF signaling. Cancer Cell. 2017;32(4):474-489.e6.

50. Lam HM, et al. Durable response of enzalutamide-resistant prostate cancer to supraphysiological testosterone is associated with a multifaceted growth suppression and impaired DNA damage response transcriptomic program in patient-derived xenografts. Eur Urol. 2019;S0302-2838(19):30449-X.

51. Kim K, et al. Identification of E2F1 as a positive transcriptional regulator for $\delta$-catenin. Biochem Biophys Res Commun. 2008;369(2):414-420.

52. Yu J, et al. Induced pluripotent stem cell lines derived from human somatic cells. Science. 2007;318(5858):1917-1920.

53. Spans L, et al. Comparative genomic and transcriptomic analyses of LNCaP and C4-2B prostate cancer cell lines. PLoS One. 2014;9(2):e90002.

54. Sharp A, et al. Androgen receptor splice variant-7 expression emerges with castration resistance in prostate cancer. J Clin Invest. 2019;129(1):192-208.

55. Yang X, et al. A public genome-scale lentiviral expression library of human ORFs. Nat Methods. 2011;8(8):659-661.

56. Robinson MD, McCarthy DJ, Smyth GK. edgeR: a bioconductor package for differential expression analysis of digital gene expression data. Bioinformatics. 2010;26(1):139-140.

57. Doench JG, et al. Optimized sgRNA design to maximize activity and minimize off-target effects of CRISPR-Cas9. Nat Biotechnol. 2016;34(2):184-191.

58. Ma J, et al. CRISPR-DO for genome-wide CRISPR design and optimization. Bioinformatics. 2016;32(21):3336-3338

59. Park RJ, et al. A genome-wide CRISPR screen identifies a restricted set of HIV host dependency factors. Nat Genet. 2017;49(2):193-203. 